\title{
The Luminosity Function of X\#Ray-selected Active Galactic Nuclei: Evolution of Supermassive Black Holes at High Redshift
}

\section{Citation}

Silverman, J. D., P. J. Green, W. A. Barkhouse, D.\#W. Kim, M. Kim, B. J. Wilkes, R. A. Cameron, et al. 2008. "The Luminosity Function of X\#Ray-selected Active Galactic Nuclei: Evolution of Supermassive Black Holes at High Redshift." The Astrophysical Journal 679 (1) (May 20): 118139. doi:10.1086/529572.

\section{Published Version}

doi:10.1086/529572

\section{Permanent link}

http://nrs.harvard.edu/urn-3:HUL.InstRepos:29921879

\section{Terms of Use}

This article was downloaded from Harvard University's DASH repository, and is made available under the terms and conditions applicable to Other Posted Material, as set forth at http:// nrs.harvard.edu/urn-3:HUL.InstRepos:dash.current.terms-of-use\#LAA

\section{Share Your Story}

The Harvard community has made this article openly available.

Please share how this access benefits you. Submit a story.

Accessibility 
The Astrophysical Journal, 679:118-139, 2008 May 20

(C) 2008. The American Astronomical Society. All rights reserved. Printed in U.S.A.

\title{
THE LUMINOSITY FUNCTION OF X-RAY-SELECTED ACTIVE GALACTIC NUCLEI: EVOLUTION OF SUPERMASSIVE BLACK HOLES AT HIGH REDSHIFT
}

\author{
J. D. Silverman, ${ }^{1}$ P. J. Green, ${ }^{2}$ W. A. Barkhouse, ${ }^{3}$ D.-W. Kim, ${ }^{2}$ M. Kim, ${ }^{4}$ B. J. Wilkes, ${ }^{2}$ R. A. Cameron, ${ }^{2}$ \\ G. Hasinger, ${ }^{1}$ B. T. Jannuzi, ${ }^{5}$ M. G. Smith, ${ }^{6}$ P. S. Smith, ${ }^{7}$ and H. Tananbaum ${ }^{2}$ \\ Received 2006 December 8; accepted 2008 January 15
}

\begin{abstract}
We present a measure of the hard (2-8 keV) X-ray luminosity function (XLF) of AGNs up to $z \sim 5$. At high redshifts, the wide area coverage of the Chandra Multiwavength Project is crucial to detect rare and luminous $\left(L_{\mathrm{X}}>\right.$ $10^{44} \mathrm{ergs} \mathrm{s}^{-1}$ ) AGNs. The inclusion of samples from deeper published surveys, such as the Chandra Deep Fields, allows us to span the lower $L_{\mathrm{X}}$ range of the XLF. Our sample is selected from both the hard $\left(z<3, f_{2-8 \mathrm{keV}}>6.3 \times\right.$ $\left.10^{-16} \mathrm{ergs} \mathrm{cm}^{-2} \mathrm{~s}^{-1}\right)$ and soft $\left(z>3, f_{0.5-2.0 \mathrm{keV}}>1.0 \times 10^{-16} \mathrm{ergs}^{-2} \mathrm{~cm}^{-1}\right)$ energy band detections. Within our optical magnitude limits $\left(r^{\prime}, i^{\prime}<24\right)$, we achieve an adequate level of completeness $(>50 \%)$ regarding X-ray source identification (i.e., redshift). We find that the luminosity function is similar to that found in previous X-ray surveys up to $z \sim 3$ with an evolution dependent on both luminosity and redshift. At $z>3$, there is a significant decline in the numbers of AGNs with an evolution rate similar to that found by studies of optically selected QSOs. Based on our $\mathrm{XLF}$, we assess the resolved fraction of the cosmic X-ray background, the cumulative mass density of SMBHs, and the comparison of the mean accretion rate onto SMBHs and the star formation history of galaxies as a function of redshift. A coevolution scenario up to $z \sim 2$ is plausible, although at higher redshifts the accretion rate onto SMBHs drops more rapidly. Finally, we highlight the need for better statistics of high-redshift AGNs at $z \gtrsim 3$, which is achievable with the upcoming Chandra surveys.
\end{abstract}

Subject headings: galaxies: active — quasars: general — surveys — X-rays: galaxies

\section{INTRODUCTION}

Our present understanding of the evolution of accreting supermassive black holes (SMBHs) over cosmic time comes from the measure of the luminosity function (i.e., the number undergoing a detectably luminous phase within a specific comoving volume as a function of luminosity and redshift) of active galactic nuclei (AGNs). Energy production through mass accretion onto SMBHs allows us to observationally identify these sites as the familiar AGNs, with quasi-stellar objects (QSOs) the most luminous example. Hence, the AGN luminosity function provides a key constraint to discern the underlying physical properties of the population (i.e., black hole mass and accretion rate distributions as a function of redshift) and thereby elucidate the mechanisms (i.e., galaxy mergers and/or self-regulated growth) that are instrumental in their formation and evolution.

To date, an enormous effort has been undertaken to measure the luminosity function over the wide range in luminosity spanned by AGNs out to high redshift. The bright end has been well established to $z \sim 5$ by optical surveys (e.g., Richards et al. 2006; Croom et al. 2004; Wolf et al. 2003) that primarily select QSOs using multicolor photometric criteria. The most dramatic feature found is the rise and fall of the comoving space density with peak activity at $z \sim 2.5$. With an unprecedented sample of over 20,000 QSOs in the 2dF QSO Redshift Survey (2QZ), Croom et al. (2004) convincingly show a systematic decrease in lumi-

\footnotetext{
1 Max-Planck-Institut für extraterrestrische Physik, D-84571 Garching, Germany.

2 Harvard-Smithsonian Center for Astrophysics, Cambridge, MA 02138.

3 Department of Astronomy, University of Illinois, Urbana, IL 61801.

4 International Center for Astrophysics, Korea Astronomy and Space Science Institute, Yusong, Taejon 305-348, Korea.

5 National Optical Astronomy Observatory, Tucson, AZ 85726-6732.

6 Cerro Tololo Inter-American Observatory, National Optical Astronomical Observatory, Casilla 603, La Serena, Chile.

7 Steward Observatory, University of Arizona, Tucson, AZ 85721.
}

nosity (pure luminosity evolution [PLE]) from $z=2$ to the present, in agreement with past surveys (e.g., Schmidt \& Green 1983; Boyle et al. 1988; Hewett et al. 1993), which find very few bright QSOs in the local universe. This fading of the luminous QSO population is thought to be due to a decrease in the mass accretion rate (e.g., Cavaliere \& Vittorini 2000) that appears to be intimately related to the order-of-magnitude decline of the cosmic star formation rate (SFR) from $z \sim 1$ to the present (Boyle \& Terlevich 1998; Franceschini et al. 1999; Merloni et al. 2004). The drop-off in the space density at $z>3$ (Osmer 1982; Warren et al. 1994; Schmidt et al. 1995; Fan et al. 2001, 2004; Wolf et al. 2003) may be indicative of either the detection of the onset of accretion onto young SMBHs or a high-redshift population that has been missed, possibly under a veil of obscuration (e.g., Alexander et al. 2005; Martinez-Sansigre et al. 2005). Excessive amounts of dust and gas may be ubiquitous in galaxies at early epochs due to an increase in the merger rate (Kartaltepe et al. 2007) that induces high SFRs (e.g., Chapman et al. 2005).

It has been evident for quite some time that optical surveys miss a significant fraction of the AGN population. They fail to find the majority of AGNs due to a steeply declining luminosity function with the low-luminosity end severely affected by host galaxy dilution. Although current optical selection techniques do show considerable improvement (Richards et al. 2005; Jiang et al. 2006), they still fail to account for many low-luminosity AGNs. Of equal significance, many AGNs (e.g., Seyfert 2 galaxies, narrow-line radio galaxies) are missed due to dust obscuration (causing their optical properties to differ from the type 1 QSOs) and can only be adequately selected in the low-redshift universe (Hao et al. 2005) based on their highly ionized, narrow emission lines. Fortunately, AGNs can now be efficiently accounted for by selection techniques in other wave bands such as the X-ray (as demonstrated in this work), radio (e.g., Wall et al. 2005), and infrared (e.g., Polletta et al. 2006). As further elaborated below, current models based on recent observations continue to attribute the bulk of the 
cosmic X-ray background (CXB), the previously unresolved X-ray emission, to these various types of obscured AGNs.

Over more than two decades, X-ray surveys have been improving and extending the known AGN luminosity function by including sources at low luminosity, with or without optical emission lines, and hidden by a dense obscuring medium. The Extended Medium Sensitivity Survey (Gioia et al. 1990) was one of the first surveys to measure the X-ray luminosity function (XLF) out to the QSO peak using a sample of just over 400 AGNs detected by the Einstein Observatory. Since the survey only probed the more luminous AGNs $\left(L_{\mathrm{X}}>10^{44} \mathrm{ergs} \mathrm{s}^{-1}\right)$ above $z=0.3$ due to the bright flux limit $\left(f_{0.3-3.5 \mathrm{keV}}>5 \times 10^{-14} \mathrm{ergs} \mathrm{cm}^{-2} \mathrm{~s}^{-1}\right)$, it was quite understandable that Maccacaro et al. (1991) and della Ceca et al. (1992) found the XLF to behave similarly to the optical luminosity function (i.e., PLE) with a decreasing space density from $z \sim 2$ to the present. The ROSAT satellite with its increase in flux sensitivity $\left(f_{0.5-2.0 \mathrm{keV}}>10^{-15} \mathrm{ergs} \mathrm{cm}^{-2} \mathrm{~s}^{-1}\right)$ enabled AGNs to be detected at lower luminosities and out to higher redshifts $(z \sim 4.5)$. Surveys ranged from the wide-area and shallow ROSAT Bright Survey $\left(f_{\lim } \sim 10^{-12} \mathrm{ergs} \mathrm{cm}^{-2} \mathrm{~s}^{-1}, 20,000 \mathrm{deg}^{2}\right.$; Schwope et al. 2000) to the deep and narrow Lockman Hole ( $f_{\text {lim }} \sim 10^{-15}$ ergs $\mathrm{cm}^{-2} \mathrm{~s}^{-1}, 0.3 \mathrm{deg}^{2}$; Lehmann et al. 2001). With a compilation of 690 AGNs from these fields and other available ROSAT surveys (Bower et al. 1996; Appenzeller et al. 1998; McHardy et al. 1998; Zamorani et al. 1999; Mason et al. 2000) that effectively fill in the parameter space of flux and area, Miyaji et al. (2000) were able to resolve $\approx 60 \%-90 \%$ of the soft CXB into point sources and generate a soft XLF that extended beyond the QSO peak. They found that the XLF departed from a simple PLE model, now well known to describe X-ray-selected samples as further elaborated below. A luminosity-dependent density evolution (LDDE) model was required due to the slower evolution rate of lower luminosity AGNs compared to that of the bright QSOs. The limited sky coverage at the faintest X-ray fluxes achievable with ROSAT prevented an accurate measure of both the faint-end slope at $z \gtrsim 1$ and the overall XLF at high redshift since only a handful of AGNs were identified above a redshift of 3 .

In the current era of Chandra and XMM-Newton, X-ray surveys are now able to detect not only AGNs and QSOs enshrouded by heavy obscuration but also those at high redshift $(z>3)$ with greatly improved statistics due to the superb spatial resolution and sensitivity between 0.5 and $10 \mathrm{keV}$ of these observatories. Previous X-ray missions such as Einstein and ROSAT as described above were limited to the soft band, which biases samples against absorption. The ASCA observatory (e.g., Akiyama et al. 2003) successfully found many nearby absorbed AGNs but lacked the sensitivity to detect the fainter sources contributing most of the 2-8 keV CXB. With deep observations of the Chandra Deep Field-North (CDF-N; Brandt et al. 2001), Chandra Deep FieldSouth (CDF-S; Rosati et al. 2002), and Lockman Hole (Hasinger et al. 2001), a large fraction, between $\sim 70 \%$ (Worsley et al. 2005) and $\sim 89 \%$ (Moretti et al. 2003), of the hard (2-8 keV) CXB has been resolved into point sources. Many of the hard X-ray sources found so far arise in optically unremarkable bright galaxies (e.g., Barger et al. 2003a; Tozzi et al. 2001), which can contain heavily obscured AGNs.

A more robust luminosity-dependent evolutionary scheme has emerged from recent measures of the XLF. With the inclusion of absorbed AGNs from Chandra and XMM-Newton surveys, lower luminosity AGNs are clearly more prevalent at lower redshifts $(z<1)$ than those of high luminosity that peak at $z \sim 2.5$. This behavior is due to the flattening of the low-luminosity slope at higher redshifts that has been well substantiated with hard
(2-8 keV) X-ray-selected surveys (Cowie et al. 2003; Ueda et al. 2003; Fiore et al. 2003; Barger et al. 2003b, 2005; Silverman et al. 2005b; La Franca et al. 2005). Using a highly complete soft (0.5-2.0 keV) band selected sample of over 1000 type 1 AGNs, Hasinger et al. (2005) have shown that this LDDE model accurately fits the data and shows a gradual shift of the peak in the comoving space density to lower redshifts with declining luminosity. This behavior may be evidence for the growth of lower mass black holes emerging in an "antihierarchical" or "cosmic downsizing" fashion while accreting near their Eddington limit (Marconi et al. 2004; Merloni 2004), or the embers of a fully matured SMBH population with sub-Eddington accretion rates. The former scenario has been substantiated by Barger et al. (2005) based on the optical luminosities of the galaxies hosting X-rayselected AGNs and by Heckman et al. (2004) using type 2 AGNs from the SDSS with the [O III] emission line luminosity as a proxy for the mass accretion rate and an estimate of the black hole mass from the $M-\sigma$ relation.

Even though there has been much progress, there are remaining uncertainties in the current measure of the XLF: (1) A significant number of X-ray sources in the recent surveys with Chandra and $X M M-N e w t o n$ are not identified. Mainieri et al. (2005) find that the peak of the redshift distribution shifts to higher values $(z \sim$ 1.2-1.5) when incorporating photometric redshifts for objects too faint for optical spectroscopy. (2) Barger et al. (2005) demonstrate that the XLF can be fitted equally well by a PLE model at $z<1.2$. These models only begin to substantially differ for low-luminosity AGNs at $z>1.5$ where AGN statistics are quite low with most being provided by the deep CDF-N and CDF-S observations. New moderate depth surveys such as the Extended Chandra Deep Field-South (E-CDF-S; Lehmer et al. 2005) and the Extended Groth Strip (EGS; Nandra et al. 2005) will provide additional AGNs at these luminosities and redshifts but await optical follow-up. (3) How does the AGN population behave at redshifts above the peak $(z \sim 2.5)$ of the optically selected QSO population? We have presented evidence (Silverman 2004; Silverman et al. 2005b) for a similar evolution of luminous X-ray-selected QSOs to those found in the optical surveys (e.g., Richards et al. 2006) with a decline in the comoving space density at $z>3$, but these AGNs are mainly type 1 . We do not expect the inclusion of luminous absorbed QSOs to drastically alter our measure of the XLF since they may be at most as numerous as the type 1 QSOs (Gilli et al. 2007). Recent radio (Wall et al. 2005) and near-infrared (Brown et al. 2006) surveys are further supporting a strong decline in the comoving density at high redshift.

In the present study, we measure the XLF of AGNs in the hard $\mathrm{X}$-ray (intrinsic $2-8 \mathrm{keV}$ ) band with an emphasis on reducing uncertainties at high redshift $(3<z<5)$. This paper is an extension to the preliminary results on the comoving space density of AGNs as reported by the Chandra Multiwavelength Project (ChaMP) survey (Silverman 2004; Silverman et al. 2005b). As previously described, these early epochs represent the emergence of the luminous QSOs and the rapid growth phase of young SMBHs. To date, the limited numbers of X-ray-selected AGNs at $z>3$ have constrained current measures (La Franca et al. 2005; Barger et al. 2005; Ueda et al. 2003) to lower redshifts. Motivated by Barger et al. (2005), we use the observed soft X-ray band for AGN selection above $z=3$ where we measure the rest-frame energies above $2 \mathrm{keV}$. Due to the rarity of luminous high-redshift AGNs, such an endeavor requires a compilation of surveys that covers a wide enough area at sufficient depths. As previously mentioned, a large dynamic range from the deep, narrow pencil beam to the wide, shallow surveys is required to measure a 
TABLE 1

AGNs Selected from Various X-Ray Surveys

\begin{tabular}{|c|c|c|c|c|c|c|c|}
\hline \multirow[b]{2}{*}{ NAME } & \multirow[b]{2}{*}{ TELESCOPE } & \multicolumn{3}{|c|}{ Hard-Band Selection $(z<3)$} & \multicolumn{3}{|c|}{ Soft-BAnd Selection $(z>3)$} \\
\hline & & $\begin{array}{c}\text { Depth } \\
\left(\text { ergs } \mathrm{cm}^{-2} \mathrm{~s}^{-1}\right)\end{array}$ & $\begin{array}{c}r^{\prime} \\
(\mathrm{mag})\end{array}$ & Number of AGNs & $\begin{array}{c}\text { Depth } \\
\text { (cgs) }\end{array}$ & $\begin{array}{c}i^{\prime} \\
(\mathrm{mag})\end{array}$ & Number of AGNs \\
\hline ChaMP. & Chandra & $2.7 \times 10^{-15}$ & 22.0 & 273 & $1.0 \times 10^{-15}$ & 22.0 & 13 \\
\hline CLASXS . & Chandra & $2.3 \times 10^{-15}$ & 24.0 & 103 & $5.6 \times 10^{-16}$ & 24.0 & 3 \\
\hline 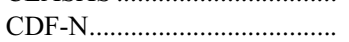 & Chandra & $6.3 \times 10^{-16}$ & 24.0 & 104 & $1.0 \times 10^{-16}$ & 24.0 & 8 \\
\hline 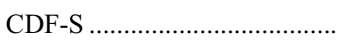 & Chandra & $6.3 \times 10^{-16}$ & 24.0 & 95 & $1.0 \times 10^{-16}$ & 24.0 & 2 \\
\hline Lockman Hole ........................... & XMM-Newton & $\ldots$ & $\ldots$ & $\ldots$ & $1.0 \times 10^{-15}$ & 24.0 & 5 \\
\hline 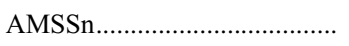 & $A S C A$ & $2.52 \times 10^{-13}$ & 22.0 & 76 & $\ldots$ & $\ldots$ & $\cdots$ \\
\hline
\end{tabular}

luminosity function that spans low and high luminosities over a broad redshift range. Currently, there are a handful of deep surveys with Chandra (i.e., CDF-N, CDF-S) and XMM-Newton (Lockman Hole) that have published catalogs with a fair sample of lowluminosity $\left(42<\log L_{\mathrm{X}}<44\right)$ AGNs out to $z \sim 5$. To provide a significant sample of more luminous AGNs ( $\left.\log L_{X}>44\right)$, the ChaMP is carrying out a wider area survey of archived Chandra fields and the CLASXS (Yang et al. 2004; Steffen et al. 2004) survey is imaging a contiguous area with nine Chandra pointings. The statistics of high-redshift AGNs are sure to improve with the anticipated results from the SWIRE/Chandra (B. J. Wilkes et al. 2008, in preparation), XBootes (Murray et al. 2005), E-CDF-S (Lehmer et al. 2005), EGS (Nandra et al. 2005), XMM/COSMOS $<$ (Hasinger et al. 2007), and Chandra/COSMOS surveys.

We organize the paper as follows: In $\S 2$ we describe the various surveys used in this analysis, including X-ray sensitivity, sky area coverage, incompleteness as a function of not only X-ray flux but also optical magnitude, and AGN selection. Our method for measuring the luminosity function is presented in $\S 3$, and the results, including best-fit analytic models, are presented in $\S 4$ for all AGN types. In $\S 5$ we address the resolved fraction of the CXB and any underrepresented source populations. We directly compare our luminosity function to that of optically selected samples in $\S 6$. Based on our luminosity function, we derive in $\S 7$ the accretion rate distribution as a function of redshift and the cumulative mass density of SMBHs. In $\S 7.1$ we compare the global mass accretion rate of SMBHs to the star formation history of galaxies out to $z \sim 5$. We end in $\S 8$ with some predictions of the numbers of high-redshift $(z>3)$ AGNs expected in new surveys that effectively enable us to extend the luminosity function to these high redshifts with accuracy. Throughout this work, we assume $H_{0}=70 \mathrm{~km} \mathrm{~s}^{-1} \mathrm{Mpc}^{-1}, \Omega_{\Lambda}=0.7$, and $\Omega_{M}=0.3$.

\section{COMPILATION OF AGNs FROM VARIOUS X-RAY SURVEYS}

The catalog of X-ray sources from the ChaMP provides the foundation for our measure of the AGN luminosity function. To this, we incorporate available catalogs from additional X-ray surveys, described below and listed in Table 1, that effectively improve the coverage of the luminosity-redshift plane. In Figure 1 we illustrate the complementarity of surveys of various depths to probe the high- and low-luminosity ends of the population; the soft band is shown to highlight the feasibility of these surveys to detect AGNs out to high redshifts $(z \sim 5)$ by taking advantage of the higher sensitivity of the soft band compared to harder $(E>2 \mathrm{keV}$ ) energy bands. As is evident, the deep surveys (i.e., CDF-N, CDF-S) are key to improving the quality of our XLF at low luminosities (e.g., $\log L_{\mathrm{X}} \sim 42$ at $1<z<3$ ), thus constraining a previously unexplored part of the luminosity function before the Chandra and XMM-Newton era. The ChaMP and CLASXS surveys, of shallower depth but of wider area, effectively supply the more luminous ( $\left.\log L_{\mathrm{X}}>44\right)$ AGNs especially at $z>1.5$ that are underrepresented in the aforementioned deep fields due to a steeply declining luminosity function. These wider area surveys are required since a sky coverage of over $1 \mathrm{deg}^{2}$ is needed to detect significant numbers of these rare, high-redshift AGNs.

We generate both a hard (2-8 keV) and soft $(0.5-2.0 \mathrm{keV})$ $\mathrm{X}$-ray-selected, flux-limited source catalog as motivated by Barger et al. (2005) to determine their rest-frame $2-8 \mathrm{keV}$ luminosity for sources with available redshifts. We thereby optimize uniformity in regards to selection across the entire compilation of data sets. The hard-band detections are used to construct a low-redshift sample at $z<3$. This enables our selection to be less affected by absorbing columns up to $N_{\mathrm{H}} \sim 10^{23.5} \mathrm{~cm}^{-2}$. The soft band allows us to take advantage of Chandra's high collecting area at low energies to detect faint, high-redshift AGNs at $z>3$. Above this redshift, intrinsic absorbing columns, if present, will have less effect on the observed flux since we are detecting X-rays at restframe energies above $2 \mathrm{keV}$. This selection technique also minimizes uncertainties induced by large $k$-corrections.

Our sample has additional selection criteria due to the dependence of source identification on optical magnitude. This is

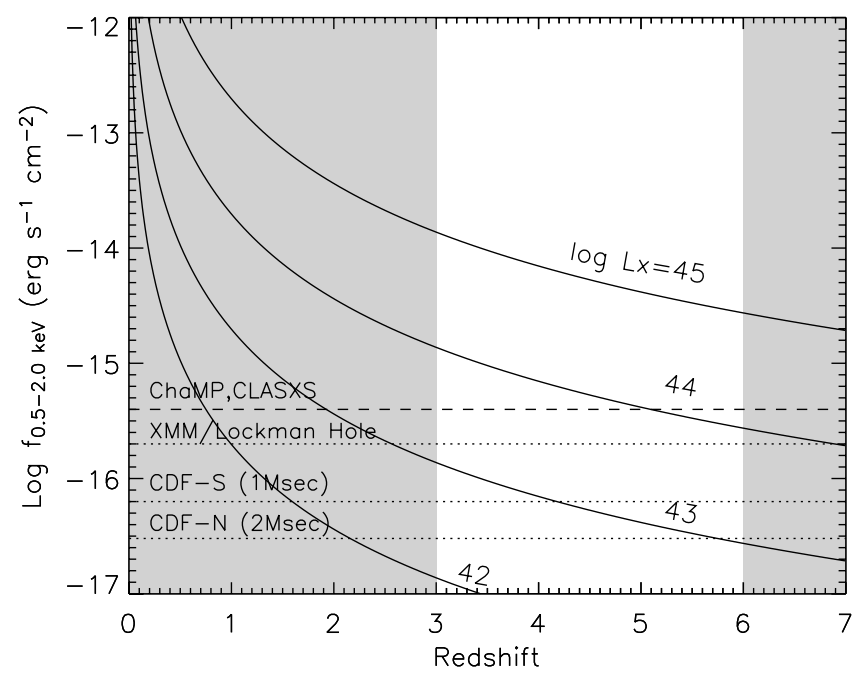

FIG. 1.- Observed flux in soft X-rays as a function of redshift for a theoretical AGN $(\Gamma=1.9)$ of luminosity (units of ergs s$\left.{ }^{-1}\right)$ as labeled. We do not consider intrinsic absorption that may be present in many AGNs. We highlight the redshift range $3<z<6$ over which an extension of the known XLF is needed. The horizontal lines mark the flux limits of various X-ray surveys capable of detecting AGNs at these high redshifts. 
clearly evident in the ChaMP by the fact that the identification of optical counterparts is currently restricted to $i^{\prime} \lesssim 23.5$ (Green et al. 2004; Silverman et al. 2005b). This is more severe $\left(i^{\prime} \lesssim 22\right)$ when considering those optical counterparts that have reliable redshifts. Additional surveys included in this study are required to have optical imaging that is available that covers a wavelength range similar to the SDSS $r^{\prime}$ and $i^{\prime}$ bands used in the ChaMP. For hardselected AGNs $(z<3)$, the $r^{\prime}$ band is sufficient to measure their optical brightness. We use the $i^{\prime}$ band for the soft-selected sample $(z>3)$ since at these high redshifts extinction due to the intervening intergalactic medium becomes significant for bandpasses at lower wavelengths. The addition of the Chandra Deep Fields not only pushes our overall catalog to fainter flux limits but improves our characterization of X-ray-bright, optically faint sources not yet identified in the ChaMP.

Our selection method based on both the X-ray and optical flux is illustrated in Figure 2. The fraction of X-ray sources with redshifts, either spectroscopic or photometric, is represented as a grayscale image determined using the adaptive binning procedure described in Silverman et al. (2005b) and a function of both X-ray flux and optical magnitude. For the hard-selected sample (top panel), we include X-ray sources from all surveys with $f_{2-8 \mathrm{keV}}>$ $2.7 \times 10^{-15} \mathrm{ergs} \mathrm{s}^{-1} \mathrm{~cm}^{-2}$ and $r^{\prime}<22.0$. To this, we add sources with fainter X-ray fluxes $\left(f_{2-8 \mathrm{keV}}<2.7 \times 10^{-15} \mathrm{ergs} \mathrm{s}^{-1} \mathrm{~cm}^{-2}\right)$ and optical magnitudes $\left(r^{\prime}>22.0\right)$ from all surveys with the exception of the ChaMP and AMSS. We set X-ray flux $\left(f_{2-8 \mathrm{keV}}>\right.$ $\left.6.3 \times 10^{-16} \mathrm{ergs} \mathrm{s}^{-1} \mathrm{~cm}^{-2}\right)$ and optical magnitude limits $\left(r^{\prime}<\right.$ $24.0)$ that effectively exclude the zone enclosed by the solid contour, where $<50 \%$ of the sources have redshifts. The same sort of two-zone selection (bottom panel) in the $f_{X} /$ opt plane occurs for the soft-selected sample with the inclusion of all sources having $f_{0.5-2 \mathrm{keV}}>1.0 \times 10^{-15} \mathrm{ergs} \mathrm{s}{ }^{-1} \mathrm{~cm}^{-2}$ and $i^{\prime}<22.0$; sources with fainter X-ray fluxes and optical magnitudes do not include ChaMP detections since currently few have identifications. For the softselected sample, we use the $i^{\prime}$ magnitudes to mitigate any flux loss due to extinction blueward of $\operatorname{Ly} \alpha$ in high-redshift AGNs. Again, we set X-ray flux $\left(f_{0.5-2.0 \mathrm{keV}}>1.0 \times 10^{-16} \mathrm{ergs} \mathrm{s}^{-1} \mathrm{~cm}^{-2}\right)$ and optical magnitude limits $\left(i^{\prime}<24.0\right)$ where $>50 \%$ of the sources in the $f_{\mathrm{X}}$ /opt plane have redshifts. The flux distribution of $\mathrm{X}$-ray sources from our above selection is shown in both the hard (Fig. 3) and soft (Fig. 4) bands. We also show the distribution of those with optical counterparts and identification (i.e., redshifts) with spectroscopic or photometric techniques. Only the CDF-N and CDF-S surveys include photometric redshifts due to the rich data sets in these fields. As is evident, we have redshifts for over $\sim 50 \%$ of the X-ray sources at all fluxes.

High X-ray luminosity becomes our single discriminant for AGN activity since many of the traditional optical AGN signatures are not present in obscured sources or may be outside spectroscopic coverage. The rest-frame $2.0-8.0 \mathrm{keV}$ luminosity (uncorrected for intrinsic absorption) is calculated by assuming a $k$-correction based on a power-law X-ray spectrum with photon index $\Gamma=1.9$ and required to exceed $10^{42} \mathrm{ergs} \mathrm{s}^{-1}$, thereby excluding any sources with X-ray emission primarily from a stellar or hot interstellar medium component. This fixed lower limit on the luminosity $\left(L_{2-8 \mathrm{keV}}>10^{42} \mathrm{ergs} \mathrm{s}^{-1}\right)$ is uniformly applied to all X-ray sources from surveys considered and yields a sample of 682 AGNs with the contribution from each survey described below and listed in Table 1. In Figure 5 we show the luminosityredshift distribution of the full sample. We now have a significant sample of 31 AGNs at $z>3$ to evaluate the luminosity function at high redshift. For the subsequent analysis, we make no attempt to subdivide the sample into the usual optical- or X-ray-based categories of AGNs (e.g., type 1/type 2, obscured/
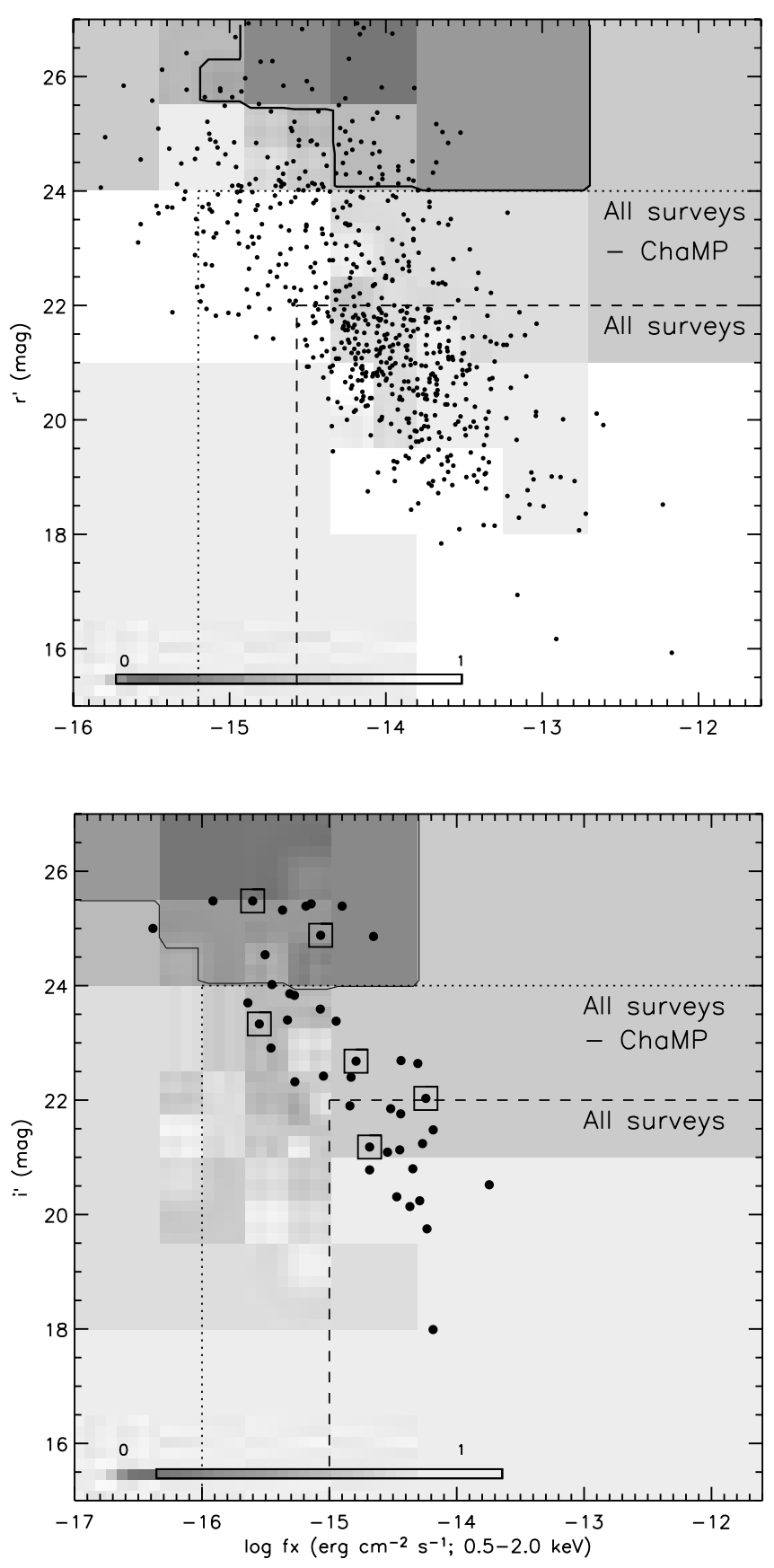

FIG. 2.-Fraction of identified (i.e., redshift is known) sources as a function of $\mathrm{X}$-ray and optical flux in the hard $/ r^{\prime}$ (top) and soft $/ i^{\prime}$ (bottom) energy bands. The gray-scale image ranges from zero (darkest) to $100 \%$ (lightest) of sources having redshifts mainly from optical spectroscopy. The AGNs are shown with a filled circle in the hard map for those at $z<3$ and soft map for those at $z>3$. In the soft-band map, those AGNs at $z>4$ are also marked by a square. The X-ray and optical flux limits for our analysis are shown by the dashed and dotted lines, respectively. Since the ChaMP survey has a reasonable degree of completeness only at brighter flux levels, we only include ChaMP AGNs that fall in the lower right region as defined by the dashed lines. The faintest limits (dotted line) are determined by the fraction of identified sources $(>50 \%)$.

unobscured). We do show for illustrative purposes in Figure 5 those AGNs that have been classified through spectroscopy or photometry as a type 1 AGN (filled symbols). It is worth highlighting the fact that almost all of the AGNs at $z>3$ may be optically unobscured as characterized by the presence of broad emission lines (FWHM $>1000 \mathrm{~km} \mathrm{~s}^{-1}$ ) in their optical spectra. Further spectroscopic follow-up with deep observations on $8 \mathrm{~m}$ 

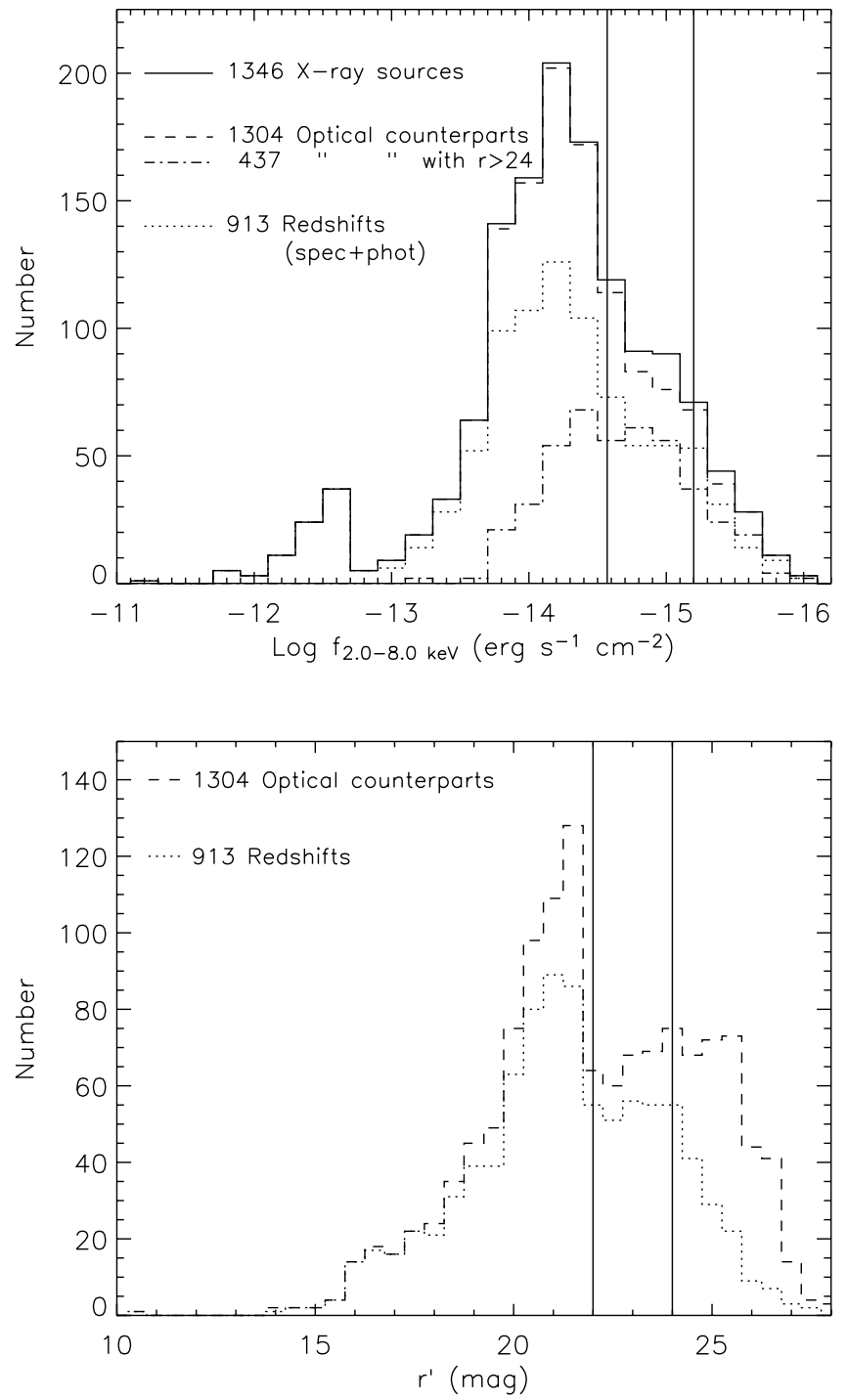

FIG. 3.-Top: X-ray flux distribution for the sources selected in the $2-8 \mathrm{keV}$ band: all X-ray sources (solid line), those with optical counterparts $\left(r^{\prime} ;\right.$ dashed line), optically faint counterparts only $\left(r^{\prime}>24\right.$; dot-dashed line), and identifications based on a spectroscopic or photometric redshift (dotted line). Bottom: Optical magnitude distribution. In both plots, the vertical lines show our chosen flux/magnitude limits, with the brighter one marking the self-imposed limit for the ChaMP survey.

class telescopes is required to adequately assess the contribution of obscured AGNs at these redshifts.

\subsection{Chandra Multiwavelength Project (ChaMP)}

The ChaMP, ${ }^{8}$ a survey of serendipitous, extragalactic X-ray sources detected in fields found in the Chandra archive, covers a large enough area at flux depths required to detect significant numbers of AGNs at high redshift. We refer the reader to the following ChaMP papers for full details concerning the X-ray analysis (Kim et al. 2004a, 2004b, 2007a, 2007b) and optical follow-up (Green et al. 2004; Silverman et al. 2005a) programs. Briefly, the ChaMP point-source catalog (Kim et al. 2007b) contains 7365 unique sources found in 149 ACIS fields with exposure times ranging from 0.9 to $124 \mathrm{ks}$ that correspond to a flux limit of $f_{0.5-8.0 \mathrm{keV}}=9 \times 10^{-16} \mathrm{ergs} \mathrm{cm}^{-2} \mathrm{~s}^{-1}$. The area coverage reaches $\sim 10 \operatorname{deg}^{2}$ at the brightest fluxes. The backbone of the optical

\footnotetext{
${ }^{8}$ See http://hea-www.harvard.edu/CHAMP/.
}
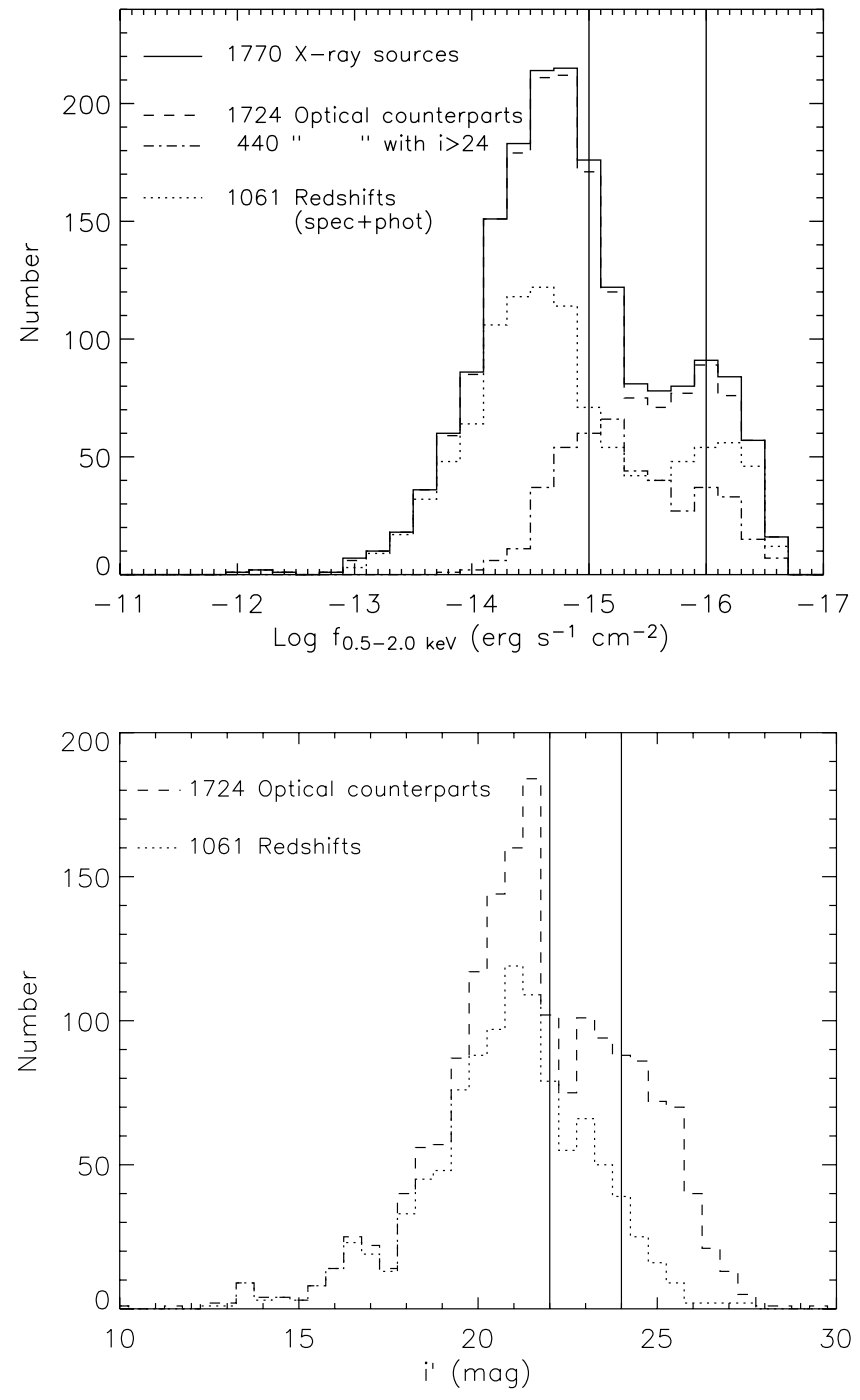

FIG. 4.- Same as Fig. 3, but for the soft $0.5-2.0 \mathrm{keV}$ and $i^{\prime}$ selected sources.

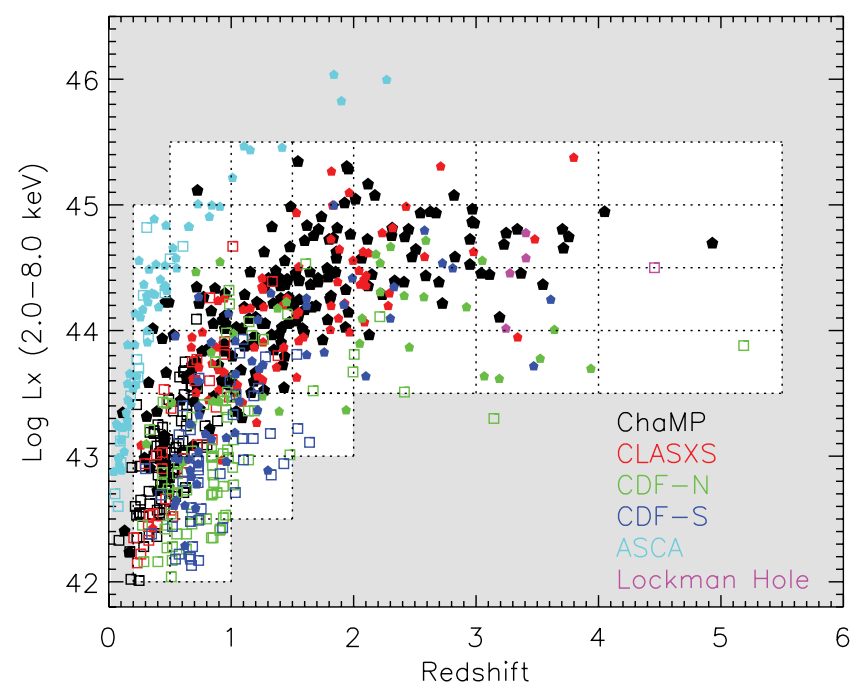

FIG. 5.- Luminosity-redshift distribution of AGNs from the X-ray surveys as labeled. Of the $682 \mathrm{AGNs}, 414$ have broad emission lines in their optical spectra shown by the solid pentagons. Open squares correspond to AGNs characterized by narrow optical emission or absorption lines. The white area highlights the regions for which our ID fraction is sufficient to measure the density of AGNs. The dashed lines denote the $L_{\mathrm{X}}-z$ bins used for the $1 / V_{a}$ method. 
TABLE 2

CHAMP FieLDS

\begin{tabular}{|c|c|c|c|}
\hline ObsID & ACIS & PI Target & $\begin{array}{c}\text { Exposure Time } \\
(\mathrm{ks})\end{array}$ \\
\hline $520 \ldots$. & I & MS 0015.9+1609 & 61.0 \\
\hline 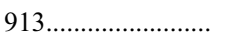 & I & CL J0152.7-1357 & 34.6 \\
\hline 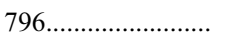 & I & SBS $0335-052$ & 47.0 \\
\hline 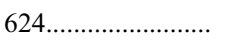 & S & LP 944-20 & 40.9 \\
\hline $902 \ldots \ldots \ldots \ldots \ldots \ldots \ldots$ & I & MS 0451.6-0305 & 41.5 \\
\hline 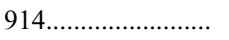 & I & CL J0542.8-4100 & 48.7 \\
\hline 377.......................... & $\mathrm{S}$ & B2 $0738+313$ & 26.9 \\
\hline $2130 \ldots \ldots \ldots \ldots \ldots \ldots \ldots$ & $\mathrm{S}$ & 3C 207 & 30.0 \\
\hline 419 & $\mathrm{~S}$ & RX J0911.4+0551 & 30.0 \\
\hline 839 & $\mathrm{~S}$ & 3C 220.1 & 20.0 \\
\hline 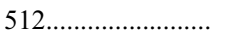 & S & EMSS $1054.5-0321$ & 75.6 \\
\hline $363 \ldots \ldots \ldots \ldots \ldots \ldots \ldots \ldots \ldots \ldots \ldots \ldots \ldots . .$. & S & PG $1115+080$ & 30.0 \\
\hline 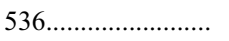 & I & MS $1137.5+6625$ & 114.6 \\
\hline 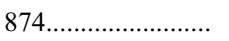 & I & $1156+295$ & 75.0 \\
\hline 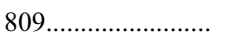 & S & Mrk 237X & 50.0 \\
\hline $541 \ldots \ldots \ldots \ldots \ldots \ldots \ldots \ldots . .$. & I & V1416+4446 & 29.8 \\
\hline 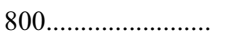 & S & CB 58 & 50.0 \\
\hline $546 \ldots \ldots \ldots \ldots \ldots \ldots \ldots \ldots \ldots \ldots \ldots \ldots \ldots \ldots$ & I & MS $1621.5+2640$ & 30.0 \\
\hline 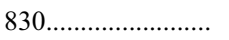 & S & Jet of 3 C 390.3 & 23.6 \\
\hline $551 \ldots \ldots \ldots \ldots \ldots \ldots \ldots$ & I & MS 2053.7-0449 & 42.3 \\
\hline $928 \ldots \ldots \ldots \ldots \ldots \ldots \ldots \ldots . .$. & S & MS 2137.3-2353 & 29.1 \\
\hline 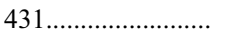 & S & Einstein cross & 21.9 \\
\hline 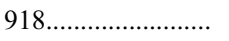 & I & CL J2302.8+0844 & 106.1 \\
\hline $861 \ldots \ldots \ldots \ldots \ldots \ldots \ldots \ldots .$. & $\mathrm{S}$ & Q2345+007 & 65.0 \\
\hline
\end{tabular}

follow-up program is a deep imaging campaign with the Cerro Tololo Inter-American Observatory (CTIO) Blanco $4 \mathrm{~m}$ and Kitt Peak National Observatory (KPNO) Mayall $4 \mathrm{~m}$ through NOAO providing images in the SDSS filters $g^{\prime}, r^{\prime}$, and $i^{\prime}$ with limiting magnitudes $(\geq 24)$ depending on the depth of the X-ray exposure. We have undertaken an optical spectroscopic follow-up program utilizing the facilities available through NOAO (i.e., WIYN/3.5 m, Blanco $4 \mathrm{~m}$, Gemini-N) and SAO (i.e., Magellan, MMT, FAST/1.2 m). Recently, we have begun a deeper spectroscopic campaign on Magellan with longer exposure times $(\sim 4 \mathrm{hr})$ and Gemini-N observations to identify the optically faint counterparts that should include the type 2 QSOs that are expected to be found in ChaMP in greater numbers (see Fig. 13 of Silverman et al. 2005a) and the most distant $(z>4)$ QSOs. In total, we have accumulated $\sim 1000$ secure spectroscopic redshifts of X-ray sources to date. In the near future, the ChaMP anticipates the release of the optical imaging and photometry for 66 fields (W. Barkhouse et al. 2008, in preparation) and spectroscopy products (P. J. Green et al. 2008, in preparation) to the community. The ChaMP is also now extending to a total of 392 fields, using Chandra Cycle 2-6 observations overlapping the SDSS (P. J. Green et al. 2008, in preparation). We have also begun to acquire near-infrared observations in the $J$ and $K_{s}$ bands for select fields using ISPI on the CTIO BLANCO $4 \mathrm{~m}$ to measure photometric redshifts and near-infrared properties for sources not accessible to optical spectroscopy.

Based on a comprehensive catalog of 1940 X-ray sources detected in 25 ChaMP fields (Table 2), we have selected a subsample of hard (2.0-8.0 keV) and soft $(0.5-2.0 \mathrm{keV})$ sources subject to X-ray and optical selection. Fields ${ }^{9}$ were chosen to have a limiting flux capable of detecting high-redshift AGNs, quality optical imaging in the $r^{\prime}$ band for the $z<3$ sample and $i^{\prime}$ for the

\footnotetext{
9 Two of the 25 fields lack $i^{\prime}$ imaging and are therefore not included in the soft-selected sample.
}
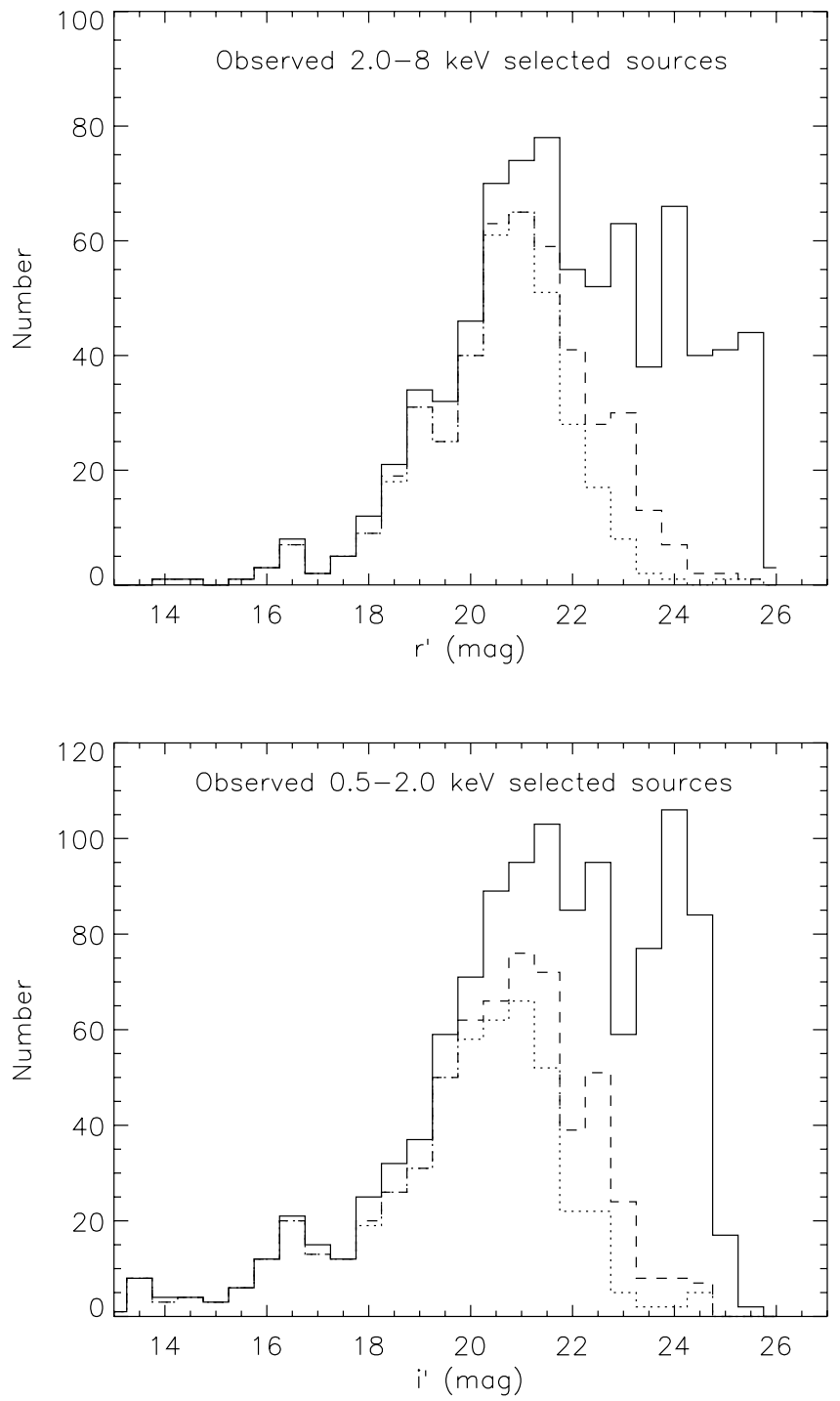

FIG. 6.-Optical magnitude distribution (solid line) of 793 X-ray-selected sources from the ChaMP in the hard sample $\left(r^{\prime} ;\right.$ top $)$ and 1125 in the soft sample $\left(i^{\prime} ;\right.$ bottom $)$. In both panels, we show those sources that have available optical spectra (dashed line) and those with reliable redshifts (dotted line). See Table 3 for a full listing of the ChaMP number statistics.

$z>3$ sample, and a substantial amount of optical spectroscopic follow-up. Sources with greater than 10 counts in their respective selection band are included. We limit our sources selected in the hard band to 392 with $f_{2-8 \mathrm{keV}}>2.7 \times 10^{-15} \mathrm{ergs} \mathrm{cm}^{-2} \mathrm{~s}^{-1}$, $r^{\prime}<22.0$ and the soft sample to 609 with $f_{0.5-2.0 \mathrm{keV}}>1.0 \times$ $10^{-15} \mathrm{ergs} \mathrm{cm}^{-2} \mathrm{~s}^{-1}, i^{\prime}<22.0$. These flux limits reflect the $\mathrm{X}$-ray and optical parameter space for which we have identified the majority $(\sim 74 \%)$ of the X-ray sources through our optical spectroscopic observations. The brightest optical counterparts were targeted for spectroscopy. This is clearly evident in Figure 6, which shows that most $(87 \%)$ of the optically bright $(<22 \mathrm{mag})$ counterparts to both soft and hard sources have measured redshifts. In Table 3 we give the number of sources for which we have obtained optical spectra and those with reliable redshifts (quality flag greater than 1). A confidence level is assigned to each redshift: $1=$ uncertain, $2=$ probably correct, $3=$ secure. Spectra with an assigned level of 1 tend to include broad emission line AGNs (BLAGNs) with a single low signal-to-noise ratio $(\mathrm{S} / \mathrm{N})$ broad emission line, whereas those with higher $\mathrm{S} / \mathrm{N}$ can be assigned a 2 or 3 depending on the number of visible spectral 
TABLE 3

ChaMP STatistics

\begin{tabular}{|c|c|c|c|c|c|c|c|}
\hline \multirow[b]{2}{*}{ X-RAY BAND } & \multirow{2}{*}{$\begin{array}{l}\text { X-Ray Flux Limit } \\
\left(\mathrm{ergs}^{-2} \mathrm{~s}^{-1}\right)\end{array}$} & \multirow[b]{2}{*}{ Optical Limit } & \multicolumn{3}{|c|}{ Numbers } & \multicolumn{2}{|c|}{ ID FRACTION (\%) } \\
\hline & & & All & Spectra & $\operatorname{IDs}^{\mathrm{a}}$ & All & Observed \\
\hline \multirow[t]{3}{*}{ 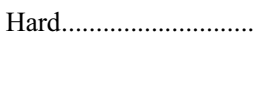 } & $2.7 \times 10^{-15}$ & None & 793 & 456 & 328 & 41 & 72 \\
\hline & $2.7 \times 10^{-15}$ & $r^{\prime}<24.0$ & 598 & 444 & 325 & 54 & 73 \\
\hline & $2.7 \times 10^{-15}$ & $r^{\prime}<22.0$ & 390 & 332 & 289 & 74 & 87 \\
\hline \multirow[t]{3}{*}{ 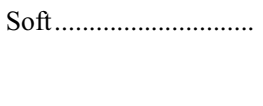 } & $4.0 \times 10^{-16}$ & None & 1125 & 624 & 435 & 39 & 70 \\
\hline & $4.0 \times 10^{-16}$ & $i^{\prime}<24.0$ & 834 & 605 & 430 & 52 & 71 \\
\hline & $4.0 \times 10^{-16}$ & $i^{\prime}<22.0$ & 512 & 429 & 373 & 73 & 87 \\
\hline
\end{tabular}

${ }^{\text {a }}$ Quality flag equal to 2 or 3.

features. Details of our spectroscopic program, including redshift measurements and quality assessment, will be presented in a future ChaMP paper (P. J. Green et al. 2008, in preparation). Spectroscopic redshifts from the ChaMP are supplemented with an additional 20 from the SEXSI (Eckart et al. 2006) and CYDER (Treister et al. 2005) surveys that have carried out observations of $\mathrm{X}$-ray sources in a subset of the ChaMP fields. We have further removed five objects identified as clusters based on their extended X-ray emission (see Barkhouse et al. 2006).

The sample of 286 AGNs with $L_{2-8 \mathrm{keV}}>10^{42} \mathrm{ergs} \mathrm{s}^{-1}$ from the ChaMP improves the statistics in select regions of the $L_{\mathrm{X}}-z$ plane and increases the number of rare types as follows: (1) type 1 AGNs or BLAGNs at $z>1.5$ detected in the $2-8 \mathrm{keV}$ band, (2) high-redshift AGNs (21 AGNs have been identified at $z>3$ [four at $z>4$ ] in the entire ChaMP program to date, roughly half the published X-ray-selected high-redshift population; optical spectra are shown in Fig. 7 for 8 of the $13 z>3$ AGNs included in this study and not yet published), and (3) $\sim 20$ galaxies with $\log L_{\mathrm{X}} \sim 43$ (Kim et al. 2006) and no strong optical emission lines (equivalent width; $W_{\lambda}<5 \AA$ ).

\subsection{Chandra Deep Field-North (CDF-N)}

The CDF-N, with an on-axis exposure time of $\approx 2 \mathrm{Ms}$, is the deepest X-ray image ever obtained (Alexander et al. 2003). Over a narrow field $\left(0.12 \mathrm{deg}^{2}\right)$, a sample of $503 \mathrm{X}$-ray sources are detected down to a limiting flux of $\approx 2.5 \times 10^{-17} \mathrm{ergs} \mathrm{cm}^{-2} \mathrm{~s}^{-1}$ in the soft band $(0.5-2.0 \mathrm{keV})$ and $\approx 1.4 \times 10^{-16} \mathrm{ergs} \mathrm{cm}^{-2} \mathrm{~s}^{-1}$ in the hard band $(2.0-8.0 \mathrm{keV})$. This is $\sim 8$ times fainter than the limiting flux of the deepest fields in the ChaMP survey. With follow-up observations in the radio through ultraviolet, this combined data set is one of the richest available for studies of the $\mathrm{X}$-ray-emitting extragalactic source population.

We select sources from the X-ray catalog (Alexander et al. 2003) that have greater than 10 counts in either band to exclude those with less certain count rates. This restricts the X-ray catalog to 330 sources in the hard band and 433 in the soft band. All fluxes are corrected for Galactic absorption using PIMMS (ver. 3.6b). ${ }^{10}$ The on-axis flux limits of this sample are $f_{2-8 \mathrm{keV}}=1.5 \times 10^{-16} \mathrm{ergs}$ $\mathrm{cm}^{-2} \mathrm{~s}^{-1}$ and $f_{0.5-2 \mathrm{keV}}=3.06 \times 10^{-17} \mathrm{ergs} \mathrm{cm}^{-2} \mathrm{~s}^{-1}$.

Barger et al. (2003a) have identified counterparts for all of the 503 X-ray sources with deep optical imaging $\left(B, V, R_{\mathrm{C}}, I_{\mathrm{C}}, z^{\prime}\right.$; Capak et al. 2004). We use photometric transformations (Fukugita et al. 1996; Smith et al. 2002) to determine $r^{\prime}$ and $i^{\prime}$ magnitudes in the SDSS photometric system for optical counterparts with $V$, $R_{\mathrm{C}}$, and $I_{\mathrm{C}}$ magnitudes. Using the 486 counterparts with magnitude in these three bands, we measure the following mean values:

\footnotetext{
${ }^{10}$ PIMMS is mostly written and maintained by Koji Mukai (http://heasarc .nasa.gov/docs/software/tools/pimms.html).
}

$\left\langle r^{\prime}-R_{\mathrm{C}}\right\rangle=0.27,\left\langle i^{\prime}-I_{\mathrm{C}}\right\rangle=0.45$, and $\left\langle R_{\mathrm{C}}-I_{\mathrm{C}}\right\rangle=0.80$. We use these to determine $r^{\prime}$ and $i^{\prime}$ when either $V, R_{\mathrm{C}}$, or $I_{\mathrm{C}}$ is undetermined. Only one X-ray source has no detections in all three bands $\left(V, R_{\mathrm{C}}\right.$, and $\left.I_{\mathrm{C}}\right)$. Any uncertainty associated with these transformations does not significantly impact our measure of the luminosity function since we mainly consider these magnitudes to measure the fraction of identified X-ray sources using wide magnitude bins. Redshifts are available for 319 (74\%) of the softband detections and 223 (68\%) of the hard-band detections with the majority $(\sim 80 \%)$ of these obtained from optical spectroscopy. The remaining identifications are derived from photometric techniques to obtain redshifts for the optically faint sources. Barger et al. (2003a) show that these are primarily at $z>1$ and have optical/near-IR emission representative of early-type galaxies. Above our self-imposed X-ray flux and optical magnitude limits (Table 1), the CDF-N sample provides 112 AGNs with $L_{(2.0-8.0 \mathrm{kev})}>10^{42} \mathrm{ergs} \mathrm{s}^{-1}$, of which 8 lie at $z>3$.

\subsection{Chandra Deep Field-South (CDF-S)}

We incorporate X-ray sources detected in the 1 Ms Chandra observation of the CDF-S (Giacconi et al. 2002) with greater than 10 counts in either the soft or hard X-ray band. Sixteen sources were removed from the main catalog that had more than one possible optical counterpart and four extended sources associated with an optical group/cluster. This results in a sample of 222 hard-selected and 256 soft-selected X-ray sources. The fluxes reported in Giacconi et al. (2002) are corrected for a negligible amount of Galactic absorption. The flux limits of this sample are $f_{2-8 \mathrm{keV}}=4.6 \times$ $10^{-16}$ ergs cm $\mathrm{cm}^{-2} \mathrm{~s}^{-1}$ and $f_{0.5-2 \mathrm{keV}}=5.6 \times 10^{-17} \mathrm{ergs} \mathrm{cm}^{-2} \mathrm{~s}^{-1}$.

We utilize the wealth of optical imaging taken of the CDF-S to determine effective SDSS magnitudes (i.e., $r^{\prime}, i^{\prime}$ ), object types, and redshifts. The $R$ magnitude, given in Giacconi et al. (2002), and the relation $\left(\left\langle r^{\prime}-R_{\mathrm{C}}\right\rangle=0.27\right)$ found using the CDF-N sources are used to determine $r^{\prime}$. We use the positions of the optical counterparts of the CDF-S sources given in Zheng et al. (2004) to search for Hubble Space Telescope Advanced Camera for Surveys (ACS) $i^{\prime}$ counterparts in the GOODS data (Giavalisco et al. 2004). For sources outside the GOODS coverage, we use the ESO imaging survey (Arnouts et al. 2001) to determine the I magnitude. In total, 301 (88\%) X-ray sources listed in Zheng et al. (2004) have an optical counterpart in the $i_{\mathrm{ACS}}$ or $I_{\mathrm{ESO}}$ band. We convert the $I_{\mathrm{ESO}}$ to $i$ using $\left\langle i^{\prime}-I_{\mathrm{ESO}}\right\rangle=0.49$, as measured from 148 sources with detections in both the GOODS and ESO imaging surveys. The ESO imaging survey provides I magnitudes for 100 of the X-ray sources not covered by the GOODS data.

Redshifts are available for 207 of the 256 soft-band sources with many (115) from spectroscopic follow-up (Szokoly et al. 2004). Multiband photometry confirms the redshifts of many of these sources (Zheng et al. 2004), including a few with uncertain 

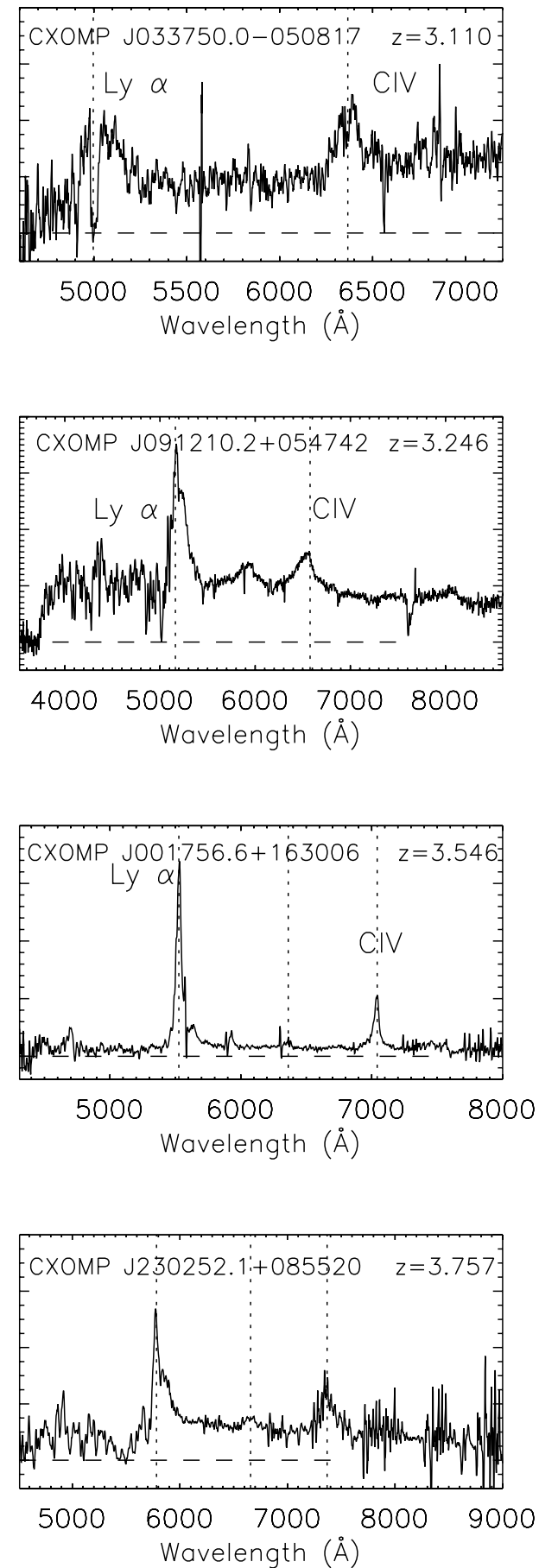
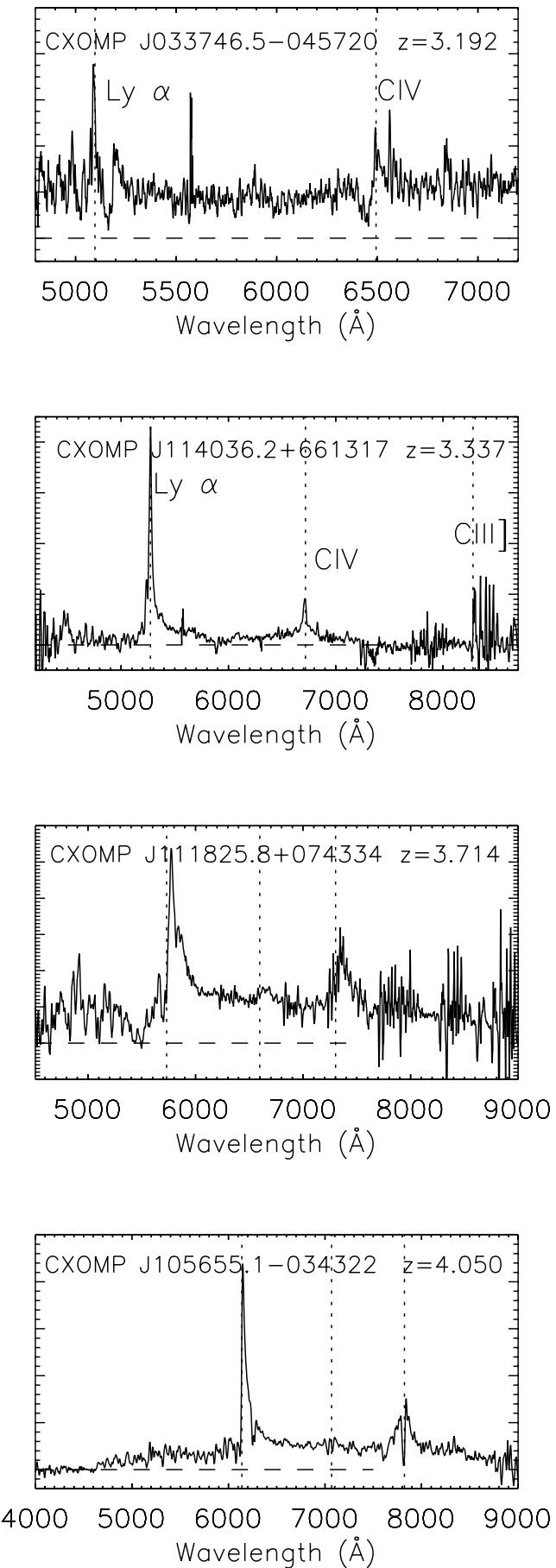

FIG. 7.-Optical spectra of AGNs at $z>3$ identified in the ChaMP. These comprise 8 of the 13 high-redshift AGNs from ChaMP included in our analysis. The remaining 5 have been published elsewhere (Silverman et al. 2002, 2003).

spectroscopic redshifts $(0<Q<2$; Szokoly et al. 2004), and identifies an additional 49 soft-selected X-ray sources. We incorporate the classifications using photometric techniques (Mainieri et al. 2002) in cases where the quality as defined in Zheng et al. (2004) is $0.5 \leq Q<1.0$. This range of quality defines those photometric redshift estimates that have similar results from at least two of the three methods. We also include photometric redshifts for those sources from the COMBO-17 survey (Wolf et al. 2004) with $R<24$ since these redshifts are fairly accurate at these brighter magnitudes. The CDF-S contributes 97 AGNs (2 at $z>$ 3 ) with $X$-ray fluxes and optical magnitudes satisfying our chosen limits (Table 1).

\subsection{Chandra Large Area Synoptic X-Ray Survey (CLASXS)}

The CLASXS survey is a contiguous mosaic of nine Chandra ACIS-I pointings in the low Galactic column region of the Lockman Hole-Northwest. The X-ray catalog (Yang et al. 2004) contains $519 \mathrm{X}$-ray sources detected in the $0.4-2.0 \mathrm{keV}$ band with a flux above $5 \times 10^{-16} \mathrm{ergs} \mathrm{cm}^{-2} \mathrm{~s}^{-1}$. To merge the sample with the ChaMP, we use the Galactic-corrected 0.5-2.0 keV fluxes listed in Steffen et al. (2004) for sources with greater than or equal to 5 counts in the respective energy selection band. We note that the conversion from counts to flux takes into account the hardness ratio of each individual source, similar to the CDF-N data. This 
can cause slight nonuniformity in our overall compilation. We assume that the area coverage shown in Figure 9 of Yang et al. (2004) in the $0.4-2.0 \mathrm{keV}$ band does not change significantly when using a slightly wider energy band. With deep optical imaging $\left(B, V, R_{\mathrm{C}}, I_{\mathrm{C}}, z^{\prime}\right)$, Steffen et al. (2004) have identified optical counterparts for $99 \%$ of the X-ray sources. As done for the CDF-N (§ 2.2) sample, we use the photometric transformations to determine $r^{\prime}$ and $i^{\prime}$ magnitudes in the SDSS photometric system for optical counterparts with $V, R_{\mathrm{C}}$, and $I_{\mathrm{C}}$ magnitudes. Using the 451 counterparts, we measure $\left\langle r^{\prime}-R_{\mathrm{C}}\right\rangle=0.23,\left\langle i^{\prime}-I_{\mathrm{C}}\right\rangle=0.43$, and $\left\langle R_{\mathrm{C}}-I_{\mathrm{C}}\right\rangle=0.37$. Only five $\mathrm{X}$-ray sources have no detections in all three $V, R_{\mathrm{C}}$, and $I_{\mathrm{C}}$ bands. Optical spectroscopic classifications are available (Steffen et al. 2004) for $52 \%$, including a significant number of optically faint $(R>24)$ counterparts. The spectroscopic classification scheme is nearly identical to the ChaMP. We group the emission-line objects (i.e., star-forming and Seyfert 2 galaxies) and absorption-line galaxies under a general "galaxy" category. Above the flux limits (Table 1) implemented for this study, there are 106 sources attributed to AGN activity based solely on their X-ray luminosity.

\subsection{XMM-Newton Lockman Hole}

We include X-ray sources found in the deep XMM-Newton observation of the Lockman Hole (Hasinger et al. 2001) to boost our sample at high redshift. We only use the soft $(0.5-2.0 \mathrm{keV})$ selected sources to add to our $z>3$ sample since the optical followup has primarily targeted soft-selected sources from the ROSAT Ultra Deep Survey (Lehmann et al. 2001). The most recent catalog (Brunner et al. 2008) contains 340 sources detected in the soft band with a limiting flux of $2 \times 10^{-16} \mathrm{ergs} \mathrm{cm}^{-2} \mathrm{~s}^{-1}$. We limit the X-ray detections to those within $12^{\prime}$ of the aim point since this region contains the majority of the sources with spectroscopic redshifts. This sample provides a fair number of AGNs at high redshift since four have been reported (Lehmann et al. 2001) at $z>3$ and one additional AGN has been identified at $z=3.244$ (G. Szokoly et al. 2008, in preparation). Optical magnitudes have been converted to SDSS magnitudes as described above ( $(2.2)$. We further limit the catalog by selecting only those sources with $\mathrm{X}$-ray flux above $f_{\mathrm{X}}>1.0 \times 10^{-15} \mathrm{ergs} \mathrm{cm}^{-2} \mathrm{~s}^{-1}$ since the fraction of identified sources at fainter fluxes is low $(<30 \%)$. Above these flux limits, there is a reasonably high degree of completeness with 59\% (69 out of 117) of the sources identified by optical spectroscopy. This field adds five AGNs at $z>3$ that also satisfy our optical magnitude selection $\left(i^{\prime}<24\right)$.

\subsection{ASCA Medium Sensitivity Survey (AMSS)}

We include AGNs from the AMSS (Akiyama et al. 2003) to tie down the bright end of the luminosity function at low redshifts. This survey presents 87 sources detected in the $2-10 \mathrm{keV}$ band with fluxes down to $3 \times 10^{-13} \mathrm{ergs} \mathrm{cm}^{-2} \mathrm{~s}^{-1}$. We convert flux to that in the $2-8 \mathrm{keV}$ band using PIMMS. We further restrict the sample to 76 AGNs with fluxes above $2.5 \times 10^{-13} \mathrm{ergs} \mathrm{s}^{-1} \mathrm{~cm}^{-2}$; this is essentially the same limit chosen by Ueda et al. (2003) to mitigate the influence of systematic effects near the flux limit of the survey. Optical photometry and source classifications are available to easily assimilate this sample of 76 AGNs into our composite catalog.

\subsection{Sky Coverage}

Each survey included in this study has published a measure of the sky area as a function of flux in both the soft and hard energy bands. To this, we add the contribution from the ChaMP (Kim et al. 2007b; Kim et al. 2007a). As described briefly in Silverman et al. (2005b) and reiterated here, a series of simulations are per-
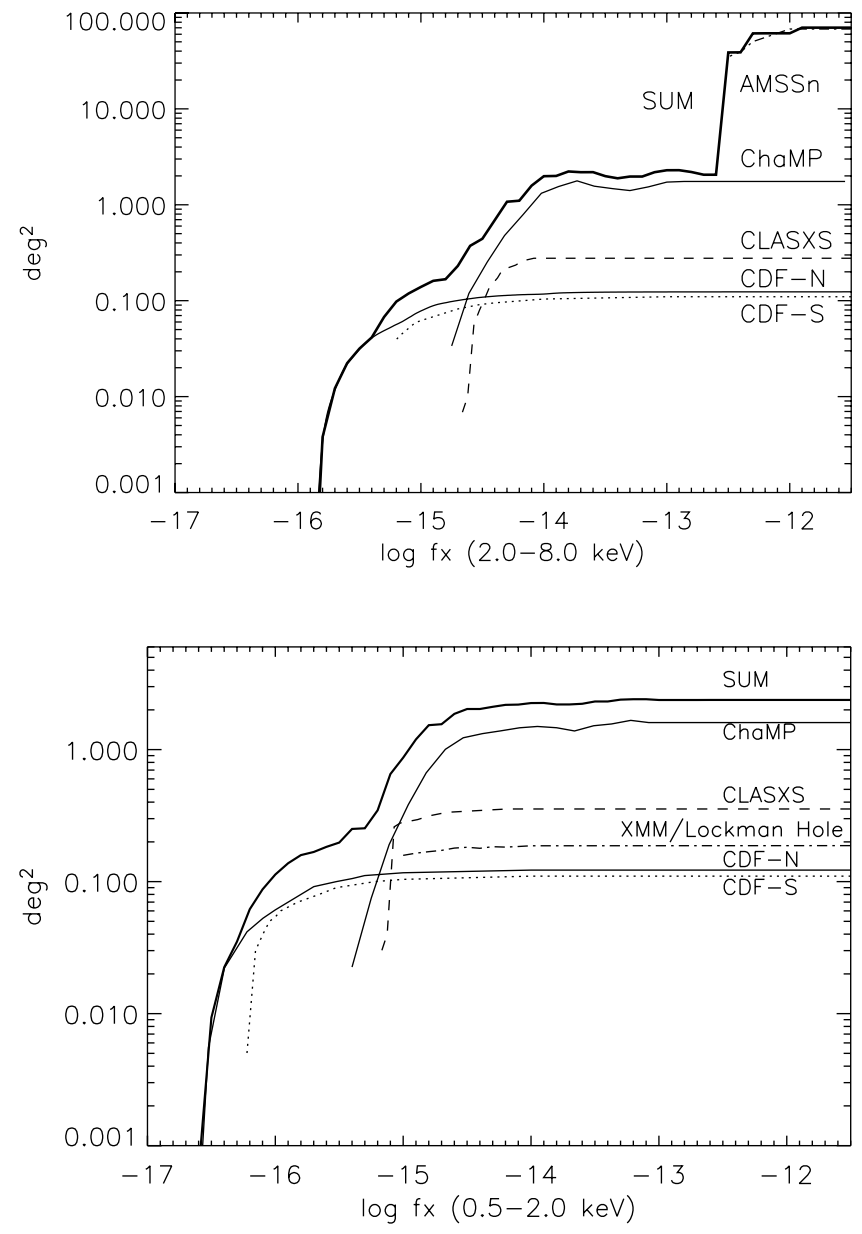

FIG. 8. - Sky area coverage as a function of X-ray flux in the hard (2.0$8.0 \mathrm{keV})$ band (top) and soft $(0.5-2.0 \mathrm{keV})$ band (bottom). The thick line is the sum of all surveys.

formed to characterize the sensitivity, completeness, and sky area coverage as a function of X-ray flux. The simulations consist of three parts: (1) generating artificial X-ray sources with MARX (MARX Technical Manual) ${ }^{11}$ and adding them to real X-ray images, (2) detecting these artificial sources by using the CIAO3.0 ${ }^{12}$ wavdetect and extracting source properties with XPIPE identically as performed for actual sources, and (3) estimating the sky area coverage as a function of flux by comparing the input and detected source properties. The simulations are restricted to specific CCDs for ACIS-I ( I0, I1, I2, and I3) and ACIS-S (I2, I3, S2, and S3). These CCDs are closest to the aim point for each observation. ACIS S4 is excluded because of its high background and noise streaks. Sources far off-axis $\left(\Theta>12^{\prime}\right)$ are excluded since the flux sensitivity is low and the point-spread function is degraded. These simulations allow determination of corrections for the source detection incompleteness at faint flux levels quantified in the first ChaMP X-ray analysis paper (Kim et al. 2004a). In Figure 8 we show the sky coverage from all surveys in the soft $(0.5-2.0 \mathrm{keV})$ and hard $(2.0-8.0 \mathrm{keV})$ bands. As shown, the ChaMP adds $\sim 2 \mathrm{deg}^{2}$ at intermediate flux levels between the narrow deep fields and the shallower, wide-area $A S C A$ surveys.

\section{MEASURING THE HARD $(2-8 \mathrm{keV})$ XLF}

We measure the differential XLF $(d \Phi / d \log L)$ expressed in equation (1), where $N$ is the number of AGNs per unit comoving

\footnotetext{
11 See http://space.mit.edu/CXC/MARX.
}

12 See http://cxc.harvard.edu/ciao. 
volume $(V)$ and $\log L_{\mathrm{X}}$ as a function of X-ray luminosity $L_{\mathrm{X}}$ and redshift $z$ :

$$
\frac{d \Phi\left(L_{\mathrm{X}}, z\right)}{d \log L_{\mathrm{X}}}=\frac{d^{2} N}{d V d \log L_{\mathrm{X}}}\left(L_{\mathrm{X}}, z\right)
$$

This function is assumed to be continuous over the range of luminosity and redshift spanned by our sample. The differential luminosity $(d \log L)$ is expressed as a logarithm (base-10) due to the 4 orders of magnitude covered by our sample.

Many methods to determine the luminosity function of extragalactic populations have been implemented (for a review see Binggeli et al. 1988). The method of choice can depend on the complexity of the selection function and/or completeness of the sample. For example, the traditional $1 / V_{a}$ method applied to a sample of quasars (Schmidt 1968) easily allows one to incorporate bivariate selection, mainly driven by incomplete optical spectroscopic identification of objects selected in another wavelength regime (i.e., radio, X-ray). Alternative techniques using binned data (e.g., Page \& Carrera 2000; Miyaji et al. 2001) have been applied to X-ray-selected AGN surveys, although they require fairly complete (i.e., optical identification and redshifts) samples. Maximum likelihood methods (Marshall et al. 1983), although model dependent, are most applicable when incorporating complex selection functions. They also mitigate effects that result from finite bin widths and irregular sampling of each bin, typically worst near the flux limit (see Fig. 1 of Miyaji et al. 2001).

We measure the luminosity function using two methods to provide further assurance of our results: (1) the $1 / V_{a}$ method and (2) a model-dependent maximum likelihood technique. The latter allows us to compare model fits and best-fit parameters to those published by Ueda et al. (2003), Barger et al. (2005), and La Franca et al. (2005). In this work, we do not consider the absorption of X-rays intrinsic to our sample, thus making no attempt to determine an $N_{\mathrm{H}}$ distribution as done in recent studies (Ueda et al. 2003; La Franca et al. 2005) since it is difficult to accurately measure absorption in AGNs at these high redshifts $(z>3)$ due to the very limited count statistics. This choice does not severely affect our results since we are using the $2-8 \mathrm{keV}$ energy band and at these high redshifts we mainly probe the bright end of the luminosity function $\left(\log L_{X}>44\right)$ that has been found to have a low fraction of absorbed sources ( $\sim 34 \%$; see Fig. 7 of Ueda et al. 2003).

\subsection{Binned $1 / V_{a}$ Method}

We estimate the XLF in fixed luminosity and redshift bins using the $1 / V_{a}$ method (Schmidt 1968; Avni \& Bahcall 1980) and our sample described in $\S 2$. For each $L-z$ bin marked in Figure 5, the value of the XLF is a sum (eq. [2]) of the contribution from each AGN falling within this specific bin:

$$
\frac{d \Phi\left(L_{\mathrm{X}}, z\right)}{d \log L_{\mathrm{X}}}=\frac{1}{\Delta \log L_{\mathrm{X}}} \sum_{i=1}^{N} C_{i} \frac{1}{V_{a, i}} .
$$

We exclude the low-luminosity bins in each redshift interval that are not well sampled at our flux limit. Our sample at $z>3$ is now sufficient to measure the luminosity function in two redshift intervals. This enables us to further probe SMBH growth at these early epochs.

We apply a correction factor $\left(C_{i}\right)$ for each AGN to account for the incompleteness in our optical spectroscopic identifications. This factor is the reciprocal of the fraction of identified sources
$\left(f_{\mathrm{ID}}\right)$ at X-ray fluxes and optical magnitudes comparable to each source, as shown by the gray-scale image in Figure 2 . The accessible volume $V_{a}$ is a function of both X-ray and optical limiting fluxes. From Hogg (1999) we measure the comoving volume for each AGN using the concordant cosmological model for $\Omega_{k}=0$ :

$$
V_{a, i}=\frac{c}{H_{0}} \int_{z_{1}}^{z_{2}\left(f_{\mathrm{X}}, f_{\mathrm{opt}}\right)} D_{L, i}^{2} \frac{\left[\Omega_{M}(1+z)^{3}+\Omega_{\Lambda}\right]^{-1 / 2}}{(1+z)^{2}} \Omega_{a}\left(L_{\mathrm{X}, i}, z\right) d z
$$

The solid angle $\Omega_{a}\left(L_{\mathrm{X}, i}, z\right)$ depends on the observed flux, which varies across the width $(d z)$ of the redshift bin, of each AGN with specific X-ray luminosity $\left(L_{\mathrm{X}, i}\right)$ to determine an accessible volume. This method does not consider evolution across the bin that may not be negligible when using large luminosity and redshift bins. Since we have a sample selected in two different energy bands, the sky coverage for AGNs at $z<3$ depends on their 2$8 \mathrm{keV}$ X-ray flux (Fig. 8, top panel) and their 0.5-2.0 keV flux for objects at higher redshifts $(z>3$; Fig. 8, bottom panel $)$. Further details on our measure of $f_{\mathrm{ID}}$ are given in Silverman et al. (2005b). We estimate $1 \sigma$ errors based on a Poisson distribution due to the small number of objects per redshift bin.

\subsection{Analytic Model Fitting}

It is highly desirable to express the luminosity function as a smooth, well-behaved analytic function in order to maximize its versatility for a wide range of uses as later illustrated. A welldefined model should be described by parameters that provide insight into the physical characteristics of the population (e.g., evolution rates). We fit our data with three different functional forms, as detailed below, over the full redshift $(0.2<z<5.5)$ and luminosity $\left(42<\log L_{\mathrm{X}}<46\right)$ range spanned by our sample. We choose models that are conventionally used in the literature in order to directly compare best-fit parameters and to extend their applicability to higher redshifts than have yet been accurately done in the hard X-ray band. As is usually the case with a data set that covers different parameter space, slight modifications of these well-utilized models are required.

To determine the values of the best-fit parameters for all models, we implement a maximum likelihood technique (Marshall et al. 1983). We minimize the following expression using the MINUIT software package (James 1994) available from the CERN Program Library:

$$
\begin{aligned}
S= & -2 \sum_{i=1}^{N} \ln \frac{d \Phi\left(L_{\mathrm{X}}^{i}, z^{i}\right)}{d \log L_{\mathrm{X}}} \\
& +2 \int_{z_{1}}^{z_{2}} \int_{l_{1}}^{l_{2}} \frac{d \Phi\left(L_{\mathrm{X}}, z\right)}{d \log L_{\mathrm{X}}} \Theta\left(f_{\mathrm{X}}, \frac{r^{\prime}}{i^{\prime}}\right) \frac{d V}{d z} d z d \log L_{\mathrm{X}} .
\end{aligned}
$$

Here $\Theta\left(f_{\mathrm{X}}\right)$ is a correction for incomplete redshift information as a function of X-ray flux as shown in the top panel of Figures 3 and 4. For this exercise, we have chosen to neglect the dependence of incompleteness as a function of optical magnitude. After each call to MINUIT the model is evaluated and compared to our total AGN sample size to determine the normalization $\left(A_{0}\right)$. We estimate the $68 \%$ confidence region for each parameter while allowing the other parameters to float freely by varying the parameter of interest until $\Delta S\left(=\Delta \chi^{2}\right)=1.0$ (Lampton et al. 1976). We note that this procedure may not accurately represent the true 
error interval since we are simultaneously fitting multiple parameters. For the normalization $\left(A_{0}\right)$, we use the Poisson error based on the sample size instead of the value of $\Delta S$.

As shown by many studies (e.g., Marshall 1987; Boyle et al. 1988), the shape of the XLF is best described as a double power law modified by a factor(s) for evolution. We first implement a "pure" luminosity evolution model (PLE; eqs. [5]-[7]) that is almost identical to that used by Richards et al. (2006) and Wolf et al. (2003). We drop the third-order term for $L_{*}$ (eq. [6]) because any incorporation of higher order evolution terms would require a significantly larger sample of X-ray-selected AGNs not yet available. In total, the model has seven free parameters $\left(A_{0}\right.$, $\gamma 1, \gamma 2, L_{0}, e 1, e 2, z_{c}$ ). Best-fit results for this model (PLE model A) are shown in Table 4 from our maximum likelihood (ML) routine:

$$
\frac{d \Phi\left(L_{\mathrm{X}}, z\right)}{d \log L_{\mathrm{X}}}=\frac{A_{0}}{\left[L_{\mathrm{X}} / L_{*}(z)\right]^{\gamma 1}+\left[L_{\mathrm{X}} / L_{*}(z)\right]^{\gamma^{2}}},
$$

with

$$
\log L_{*}=\log L_{0}+e 1 \xi+e 2 \xi^{2}
$$

and

$$
\xi=\log \left(\frac{1+z}{1+z_{c}}\right) .
$$

We are motivated to use a model that has more flexibility to fit our data, especially at low $L_{\mathrm{X}}$. A modified "PLE" model (eqs. [5]-[7]) recently presented in Hopkins et al. (2007) is quite appropriate. This model adds two additional free parameters, a redshift dependence to the spectral indices of the double power law. In our case, we choose to incorporate this feature only for the low-luminosity slope (eq. [8]) that evidently changes with redshift. Best-fit results for this model (mod-PLE/model D) are shown in Table 4 from our ML routine:

$$
\gamma 2=(\gamma 2)_{0}\left(\frac{1+z}{1+z_{c}}\right)^{\alpha} .
$$

Third, we implement an LDDE model (eqs. [9]-[11]; note that $L_{*}=L_{0}$ here) model that has been useful in quantitatively describing the shape and evolution of the luminosity function of X-ray-selected AGNs in not only the hard band (Ueda et al. 2003; La Franca et al. 2005) but also the soft band (Hasinger et al. 2005) as well. Miyaji et al. (2000) introduced a slight variant of this LDDE model to fit the AGN sample from a compilation of ROSAT surveys of varying depth and area coverage. Using this model, they were able to provide the first evidence that a luminosity-dependent evolution scheme is required for X-rayselected samples:

$$
\frac{d \Phi\left(L_{\mathrm{X}}, z\right)}{d \log L_{\mathrm{X}}}=\frac{d \Phi\left(L_{\mathrm{X}}, 0\right)}{d \log L_{\mathrm{X}}} e\left(z, L_{\mathrm{X}}\right),
$$

where

$$
e\left(z, L_{\mathrm{X}}\right)= \begin{cases}(1+z)^{e 1}, & z \leq z_{c} \\ e\left(z_{c}\right)\left(\frac{1+z}{1+z_{c}}\right)^{e 2}, & z>z_{c}\end{cases}
$$

and

$$
z_{c}\left(L_{\mathrm{X}}\right)= \begin{cases}z_{c}^{*}\left(L_{\mathrm{X}} / L_{a}\right)^{\alpha}, & L_{\mathrm{X}} \leq L_{a}, \\ z_{c}^{*}, & L_{\mathrm{X}}>L_{a} .\end{cases}
$$

In terms of model parameters, we aim to extend the applicability of this model out to $z \sim 5$ with a reevaluation of the value of $e 2$ that describes the evolution rate beyond the cutoff redshift $z_{c}$. From our preliminary measure of the comoving space density of AGNs (Silverman et al. 2005b), we expect this value to differ sharply from other studies (Ueda et al. 2003; La Franca et al. 2005 ) with a much stronger negative evolution rate that is similar to those found in optical QSO studies (e.g., Warren et al. 1994; Schmidt et al. 1995; Wolf et al. 2003; Richards et al. 2006). In addition, the values of $\alpha$ and $z_{c}^{*}$ may differ due to our larger AGN sample at high redshift. To further simplify this complex model, we fix $L_{a}$ to the value used by Ueda et al. (2003). In total there are eight free parameters $\left(A_{0}, \gamma 1, \gamma 2, L_{0}, e 1, e 2, z_{c}^{*}, \alpha\right)$. Best-fit results for this model (LDDE models $\mathrm{B}$ and $\mathrm{C}$ ) are shown in Table 4 from our ML routine.

\section{HARD XLF: RESULTS}

\section{1. $1 / V_{a}$ Method}

Our measure of the XLF, using methods detailed in the previous section, is presented in Figure 9 for seven redshift intervals with bins of finite width in luminosity. We show smooth analytic model curves (see following section for details), including that evaluated at $z=0$ (dashed line), to aid in our visualization of the evolution with redshift and luminosity. First, we see that the shape of the XLF up to $z \sim 2$ is well approximated by the familiar double power law with a steeper bright-end slope. The shape at higher redshifts is less constrained due to the limited statistics especially below the break or knee (i.e., characteristic luminosity dividing the faint- and bright-end slopes). To first order, there is an overall shift of the XLF to higher luminosities as a function of increasing redshift (i.e., pseudo-PLE) up to $z \sim 3$ as reported by many studies in various wave bands over the past few decades. There is a decline in either luminosity or space density (Barger et al. 2005; Hasinger et al. 2005; Silverman et al. $2005 \mathrm{~b}$ ) at higher redshifts similar to the behavior seen in optical (e.g., Fan et al. 2001; Wolf et al. 2003; Richards et al. 2006) and radio-selected (Wall et al. 2005) samples. Our data preclude us from distinguishing these two modes of evolution at $z>3$. Second, we see that there is evidence for a flattening of the faint-end slope with increasing redshift most noticeable by comparing the data points with the $z=0$ model (dashed line) in the redshift interval $1.0<z<1.5$. The need to utilize more complex models (i.e., LDDE) when dealing with X-ray-selected samples (Hasinger et al. 2005; Ueda et al. 2003; Miyaji et al. 2000) is primarily due to this behavior.

In Figure 10 we compare our binned measure of the XLF to the best-fit PLE (Barger et al. 2005) and LDDE (Ueda et al. 2003; La Franca et al. 2005) models to check for consistency at $z<3$. Barger et al. (2005) report that a PLE model best represents the data at $z<1.2$, with characteristic parameters including evolution rates similar to optically selected samples (e.g., Croom et al. 2004). As shown in the two low-redshift panels (top; $z<1.5$ ), it is apparent that the PLE model provides an adequate representation of the shape and evolution of the population. As previously mentioned, a flattening of the faint-end slope, most evident at $1.0<z<1.5$ (top middle panel), is apparent and cannot be accounted for with a PLE model. Our sample does not provide enough AGNs with luminosities below the knee and $z>2$ (top 
TABLE 4

Hard XLF Best-Fit Model Parameters

\begin{tabular}{|c|c|c|c|c|c|c|c|c|c|c|c|}
\hline Model & Type & $\log A_{0}$ & $\gamma 1$ & $\gamma 2$ & $\log L_{0}$ & $e 1$ & $e 2$ & $z_{c}$ & $\log L_{a}$ & $\alpha$ & $\begin{array}{l}\text { Redshift } \\
\text { Range }\end{array}$ \\
\hline A & PLE & $-5.332 \pm 0.015$ & $2.80 \pm 0.20$ & $0.77 \pm 0.05$ & $44.92 \pm 0.10$ & $1.69 \pm 0.12$ & $-0.87 \pm 0.6$ & $1.9^{\mathrm{a}}$ & & & $0.2<z<3.0$ \\
\hline В & LDDE & $-6.077 \pm 0.015$ & $2.15_{-0.12}^{+0.42}$ & $1.10 \pm 0.13$ & $44.33 \pm 0.10$ & $4.00 \pm 0.28$ & $-1.5^{\mathrm{a}}$ & $1.9^{\mathrm{a}}$ & $44.6^{\mathrm{a}}$ & $0.317_{-0.027}^{+0.020}$ & $0.2<z<3.0$ \\
\hline С & LDDE & $-6.163 \pm 0.015$ & $2.15^{b}$ & $1.10^{\mathrm{b}}$ & $44.33^{\mathrm{b}, \mathrm{c}}$ & $4.22_{-0.27}^{+0.20}$ & $-3.27_{-0.34}^{+0.31}$ & $1.89_{-0.06}^{+0.14}$ & $44.6^{\mathrm{a}}$ & $0.333 \pm 0.013$ & $0.2<z<5.5$ \\
\hline $\mathrm{D}$ & Mod-PLE & $-5.238 \pm 0.015$ & $2.76_{-0.19}^{+0.21}$ & $0.42 \pm 0.05$ & $44.88_{-0.29}^{+0.11}$ & $-0.60_{-0.14}^{+0.15}$ & $-8.18 \pm 0.55$ & 2.0 (fixed) & $\ldots$ & $-1.04_{-0.12}^{+0.11}$ & $0.2<z<5.5$ \\
\hline
\end{tabular}

a Fixed parameters to those best-fit values from Ueda et al. (2003).

Fixed to the best-fit value in model $\mathrm{B}$

${ }^{c}$ Note that for this case $L_{*}=L_{0}$. 

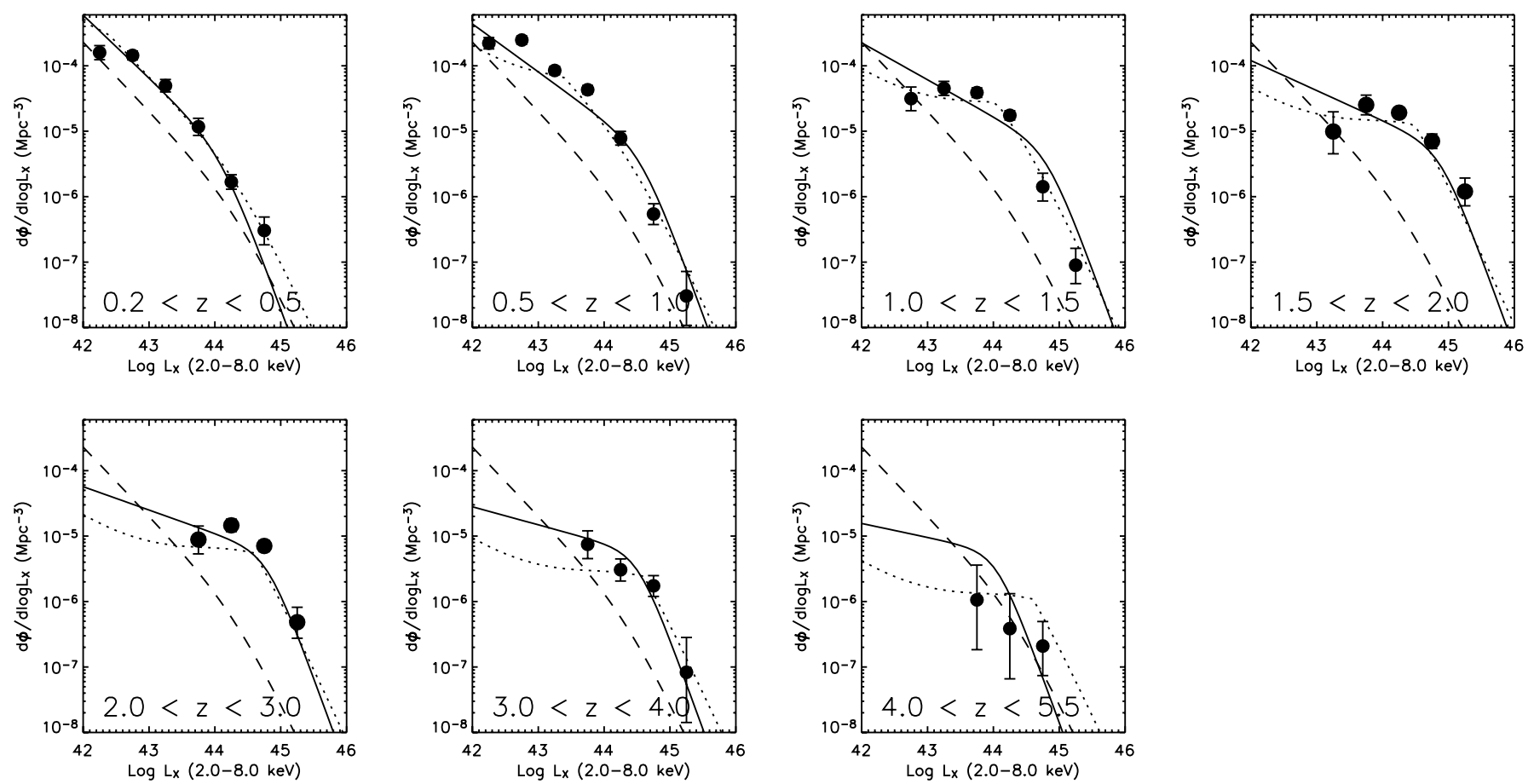

FIG. 9.-XLF. The results from the binned $1 / V_{a}$ method are given by filled circles with $1 \sigma$ errors. Our best-fit analytic models using an unbinned maximum likelihood method are shown by the lines (solid line: mod-PLE model D; dotted line: LDDE model C). The dashed line is our LDDE model C evaluated at $z=0$.
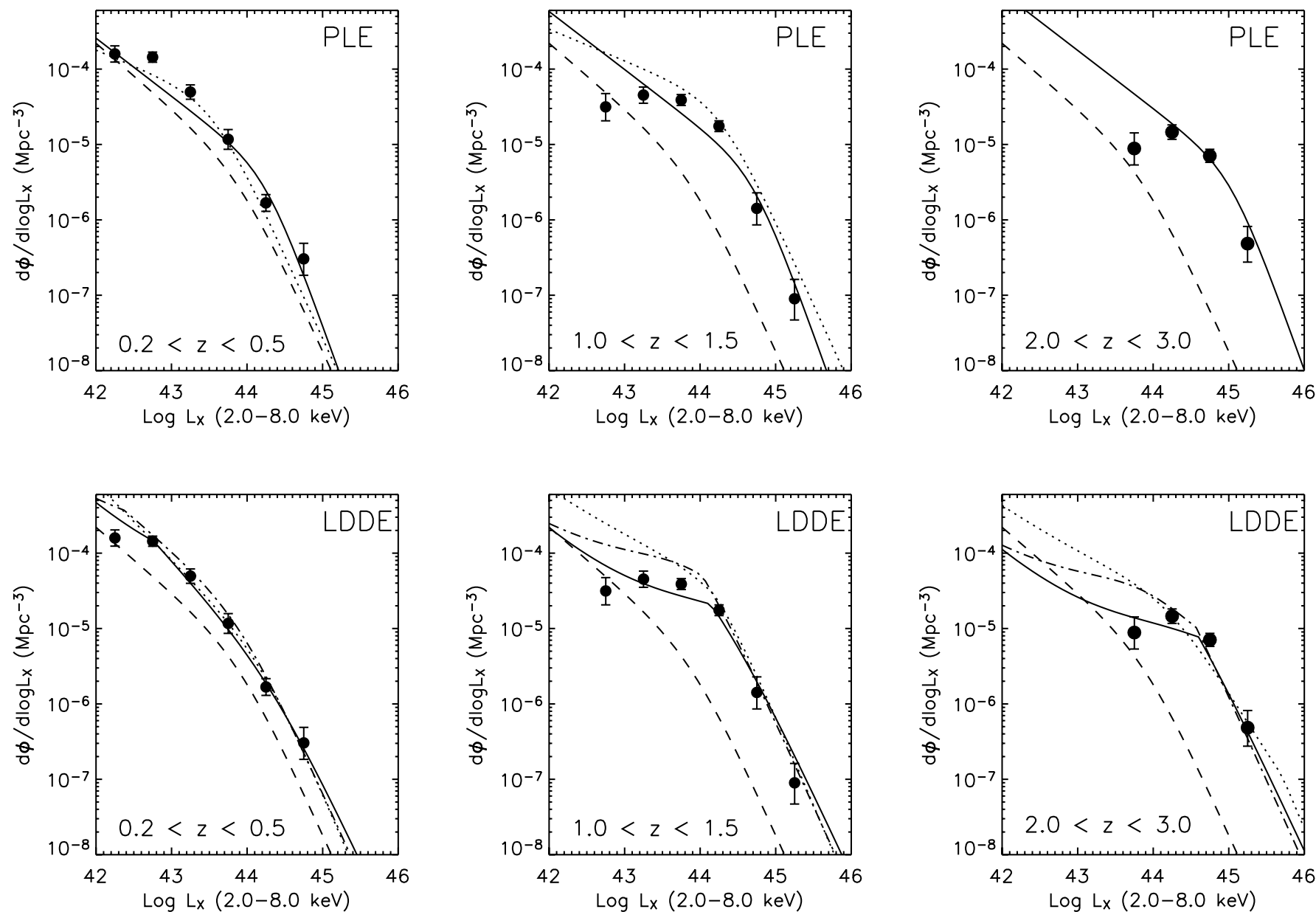

FIG. 10.-Comparison of our binned XLF ( $1 / V_{a}$ method; filled circles) and analytic model fits at $z<3$ to recently published models. Top: ChaMP PLE model A (solid

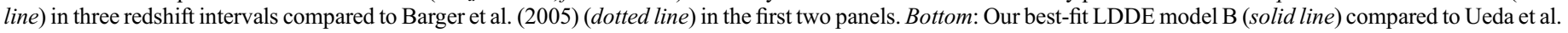
(2003) (dot-dashed line) and La Franca et al. (2005) (fit 4; dotted line). The dashed line is the Ueda model evaluated at $z=0$ in all panels. 
right panel) to constrain the faint-end slope; this an unfortunate consequence of our optical magnitude limits and incomplete spectroscopic follow-up. In the bottom panels, we compare our data with the more complex LDDE of Ueda et al. (2003) and La Franca et al. (2005). In general, the data agree well with both models, which are nearly equivalent especially at high luminosities. It is worth noting that there is some slight uncertainty in the slope and normalization at the faint end $\left(\log L_{\mathrm{X}}<44\right)$ that may be due to X-ray sources that have not been included due to our optical magnitude selection. We note that the lowest luminosity point in each of the panels is clearly affected by the fact that AGNs do not entirely fill each $L_{X}-z$ bin (see Fig. 5) that falls on or near our flux limits. An accurate measure of the faint-end XLF is beyond the scope of this paper and requires more complete samples.

As previously mentioned, we see a significant drop in the LF above $z \sim 3$ in either normalization or characteristic luminosity over two redshift intervals (Fig. 9). This behavior is similar to the decline seen in the soft and broad Chandra bands that we reported in Silverman et al. (2005b). With this new sample, we can further constrain the slope and normalization of the XLF at these high redshifts although still larger samples are required, especially at $z>4$, where only four AGNs are included in this analysis. Between $3<z<4$, our sample of 26 AGNs does show a significant decline from the peak activity at $2<z<3$. To check the integrity of our method that utilizes the observed soft X-ray band to measure the XLF above $z=3$, we have measured the XLF in the redshift interval $3<z<4$ using the hard band as done at lower redshifts. These two measures, shown in Figure 11, agree within $\sim 1 \sigma$ for each data point.

It is useful to illustrate how the ChaMP sample improves the measure of the hard XLF. In Figure 12 we display the XLF as measured with the $1 / V_{a}$ method in the three highest redshift intervals. In the top row the entire sample is used, while the bottom row includes all AGNs with the exception of those from the ChaMP. The sample size is given for each data point. For reference, the best-fit LDDE model of Ueda et al. (2003) (solid line) clearly demonstrates the difference in our results at $z>3$. First, it is evident that a similar decline in the normalization of the XLF is seen with and without the ChaMP data at $z>3$. The XLF in the bottom panels is in agreement with the behavior of the XLF reported by Barger et al. (2005) using practically similar samples. The ChaMP does improve on the accuracy of the highredshift XLF by boosting the overall numbers, including those in specific regions of the $L_{\mathrm{X}}-z$ plane having limited statistics: (1) the bright-end $\left(\log L_{\mathrm{X}}>45\right)$ slope at $2<z<3$ is now further constrained, (2) measurement errors in the redshift interval $3<z<$ 4 are effectively reduced, and (3) the numbers of $z>4$ AGNs are doubled, with an additional data point at higher luminosities ( $\log L_{\mathrm{X}} \sim 44.8$ ) that further constrains the slope. Results based solely on the ChaMP sample are presented in both Silverman et al. (2005b) and Silverman (2004).

As frequently represented, the XLF can also be plotted as a function of redshift for AGNs within a fixed luminosity interval. We show in Figure 13 the comoving space density for three luminosity ranges. The lowest luminosity range is limited to $z<1$ due to the lack of X-ray sensitivity at higher redshifts. For higher luminosities ( $\log L_{X}>43.5$ ), our sample provides suitable numbers of AGNs out to $z \sim 5$. We clearly see an increase in the number of AGNs with redshift compared to the local universe followed by a decline beyond a peak whose redshift is dependent on luminosity. The most luminous AGNs $\left(L_{X}>44.5\right)$ peak at a redshift around $z \sim 2$, similar to the optical surveys, while the lower luminosity AGNs are most prevalent at $z \sim 1.0$. We have plotted the measurements using the observed hard band (open

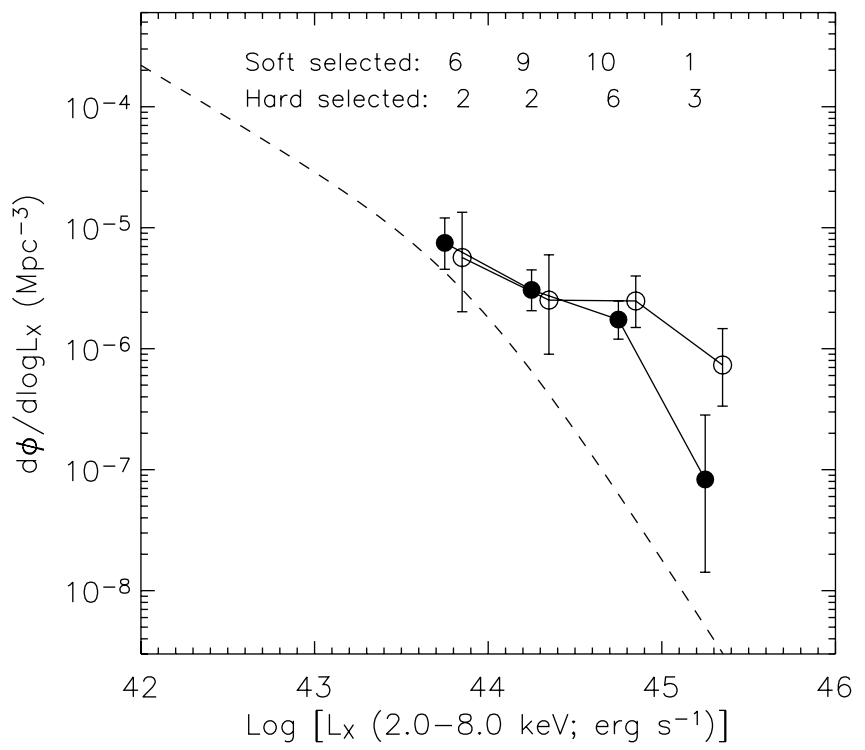

FIG. 11.-XLF in the redshift range $3<z<4$ for AGNs selected in the soft band ( filled circles) and hard band (open circles). The numbers of AGNs in each bin are reported at the top. The similarity of these XLFs and the improved statistics in the soft band illustrate our justification for using an energy-dependent selection function. For reference, we show the analytic model from Ueda et al. (2003) at $z=0$ (dashed line).

symbols) to show that the decline at $z>3$ is not due to a significant population of high-redshift sources not detected in the soft band. These results are in agreement with similar studies (Ueda et al. 2003; Barger et al. 2005; La Franca et al. 2005; Hasinger et al. 2005) with an improvement in constraints at $z>3$ for the hard XLF.

We note that X-ray sources without redshifts contribute to the uncertainty in both our XLF and space density. While those that were never observed spectroscopically might be expected to be similar in properties (e.g., in distributions of object type and redshift), the (significantly smaller) number of objects that we have observed spectroscopically without achieving a redshift may differ, which could bias our simple fractional incompleteness correction scheme. To illustrate their possible influence, we measure maximum values of the comoving space density (Fig. 13) by placing all possible unidentified objects into each redshift bin (Barger et al. 2005; Silverman et al. 2005b). We see that the highest redshift bins $(z>3)$ are most susceptible to additional uncertainty if the unidentified objects have a redshift distribution dissimilar to our AGN sample.

\subsection{Analytic Model Fitting}

Before we present a global fit over all redshifts and luminosities spanned by our sample, we verify that our method as described in $\S 3.2$ is robust. This is done by fitting our data with well-known models over a similar range of redshift and then comparing the resulting best-fit parameters to published values. First, we ran our ML routine over a redshift interval of $0.2<$ $z<3.0$ using a PLE model, similar to Barger et al. (2005), and an LDDE model with fixed parameters $\left(e 2, L_{a}\right.$, and $\left.z_{c}\right)$ having the same value as given in Ueda et al. (2003). Specifically for the LDDE model, we are interested in comparing the values of our free parameters descriptive of the shape $(\gamma 1, \gamma 2)$, evolution $(e 1)$ below the cutoff redshift $\left(z_{c}\right)$, and strength $(\alpha)$ of the dependence of $z_{c}$ on luminosity.

In Figure 10 we plot the best-fit results from our ML routine (top panel: PLE model A; bottom panel: LDDE model B; see 

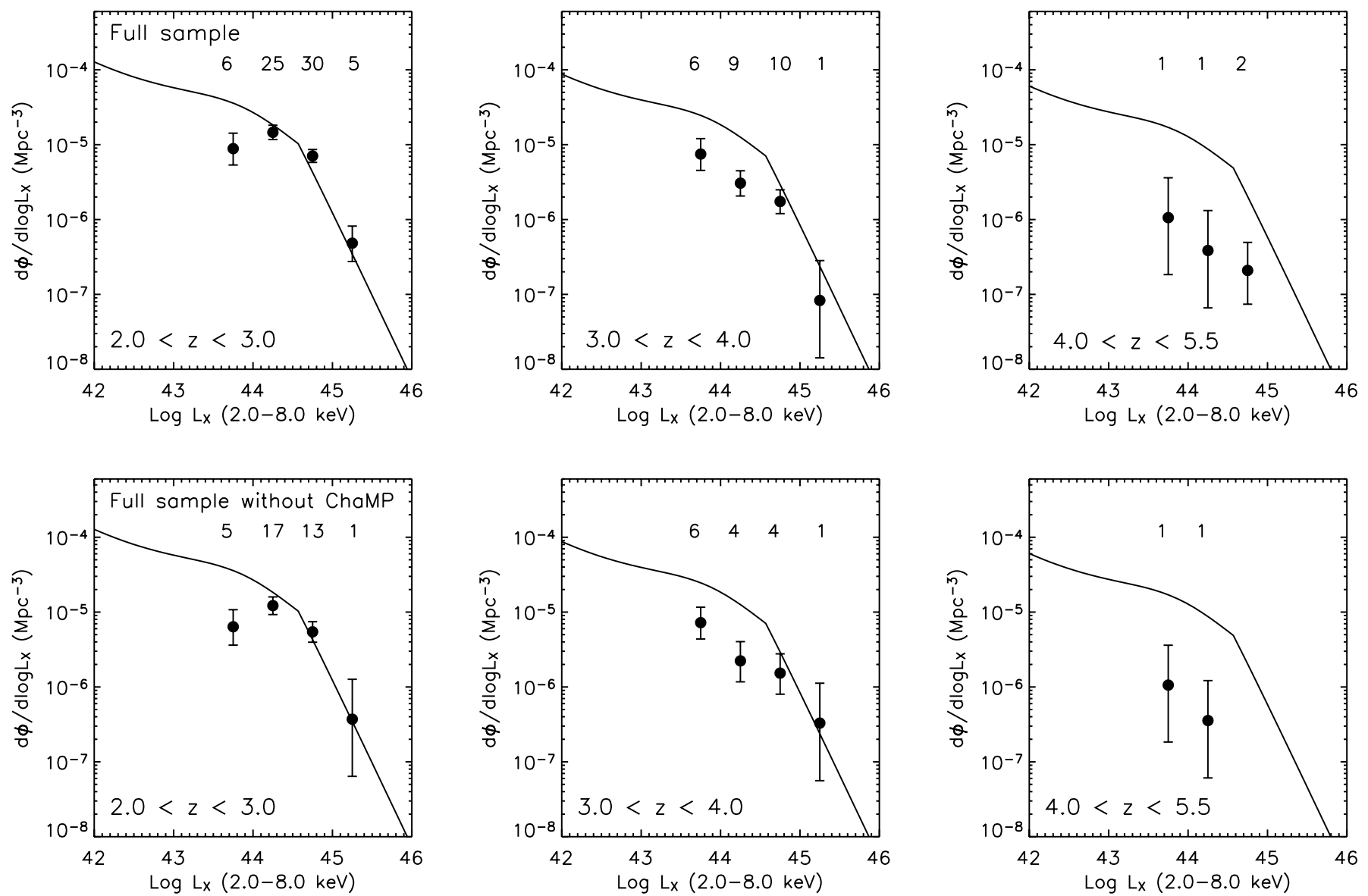

Fig. 12.-Comparison of the high-redshift XLF between the full sample (top) and that excluding the ChaMP AGNs (bottom). The data points are the same as in Fig. 9. The solid line is the best-fit LDDE model of Ueda et al. (2003) that exemplifies our differences at high redshift. The number of AGNs per luminosity and redshift bin is shown above their respective data point.

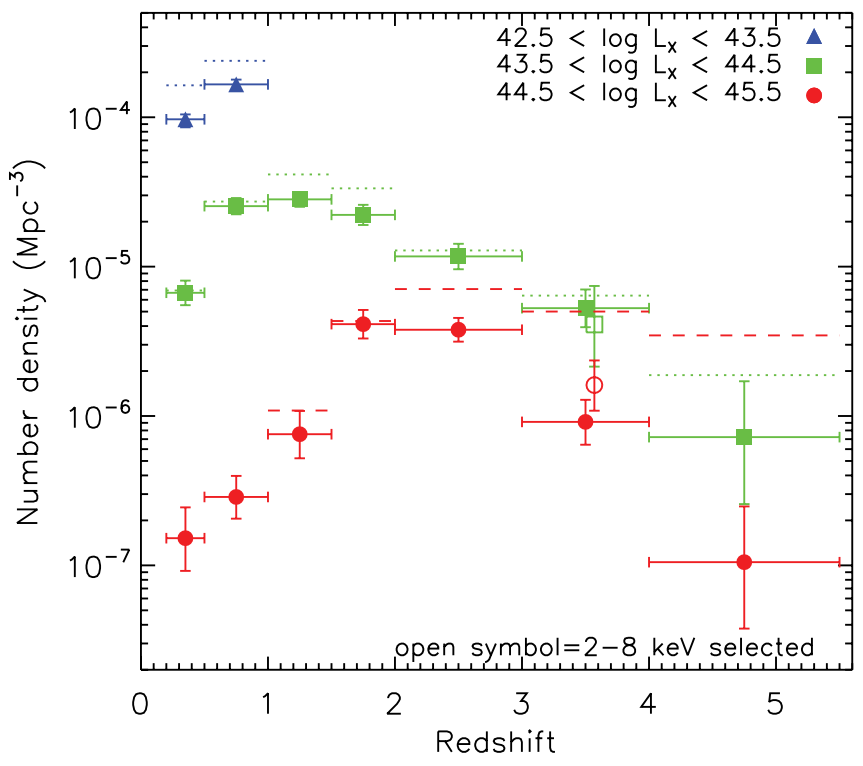

FIG. 13.-Comoving space density for three luminosity ranges using the $1 / V_{a}$ method. The open symbols show the measured values using the observed hard band. The lines (dotted and dashed) illustrate the maximum uncertainty by assuming that all possible unidentified objects fall in their respective bins.
Table 4 for actual best-fit parameters) and compare to published results (Barger et al. 2005; La Franca et al. 2005; Ueda et al. 2003) at $z<3$. Three redshift intervals are shown in each case. Our PLE model (top panel) fit, in general, agrees with that of Barger et al. (2005) at $z<1.5$. Our fit at the faint end for $z>1$ does not smoothly transverse the binned data points and thus does not adequately represent the slope below the break. Overall, there is a good agreement between our LDDE model (B; bottom panel) and that of Ueda et al. (2003) and La Franca et al. (2005) with some minor differences at the faint end that are worth noting. Below the knee of the luminosity function, our best-fit model falls below that of these two aforementioned measures. As previously noted, this may be a result of our optical magnitude limits that remove a significant fraction of the hard and soft X-ray sources above our chosen X-ray limits. This may explain (1) our lower normalization $\left(A_{0}\right)$, although sensitive to the values of the other parameters, as compared to both the Ueda and La Franca models, and (2) the differences between the faint-end slope at $z>1$. The evolution factor $(e 1)$ and power-law index for the luminositydependent cutoff redshift $(\alpha)$ are within the $1 \sigma$ errors reported by Ueda et al. (2003). Given these small discrepancies and the limitations of the data, we conclude that all three models are equally valid at $z<3$.

We aim to extend the fit of the LDDE model to higher redshift $(z>3)$. This has been attempted by La Franca et al. (2005) although with a smaller sample at these redshifts that may also be biased by cosmic variance since more than half (five of nine) of the high-redshift AGNs are found in the limited area coverage of 
the CDF-S. Since the size our sample is also restrictive at these redshifts (31), we need to reduce the number of free parameters. To do so, we fix those that are highly constrained at lower redshift: $\gamma 1, \gamma 2, L_{0}$, and $L_{a}$. The cutoff redshift $\left(z_{c}\right)$, evolution parameter above the cutoff redshift $(e 2)$, and $\alpha$ are free to vary. The most likely fit over the full redshift range $0.2<z<5.5$ is shown in Figure 9 (dotted line), with the values of each of the parameters given in Table 4 (model C). First, there is agreement with the binned values from the $1 / V_{a}$ method with some exceptions elaborated further below. The main improvement, as compared to published hard XLFs, is that the fit requires a much stronger evolution $\left(e 2=-3.27_{-0.34}^{+0.31}\right)$ above the cutoff redshift than previously found by Ueda et al. (2003; $e 2=-1.5)$ and La Franca et al. $(2005 ; e 2=-1.15)$. Our value is more constrained as evident by the $1 \sigma$ errors that are $\sim 2$ times smaller than those reported by La Franca et al. (2005).

There is a slight discrepancy between the LDDE fit and the binned data from our $1 / V_{a}$ method worth highlighting. The strong evolution (e2) above the cutoff redshift from our fit causes the faint-end slope $\left(\log L_{\mathrm{X}}<44.5\right)$ to flatten a bit more rapidly with increasing redshift than shown by the binned data. This is evident in the $2<z<3$ and $3<z<4$ redshift bins in Figure 9, with the lowest luminosity points falling above the LDDE fit (dotted line), although the statistics are too low to definitely make such claims. This may just illustrate the difficulties in accurately modeling the XLF with underlying complexities in addition to the abrupt change from positive to negative evolution. It is also worth recognizing that the faint-end slope of the luminosity function at these high redshifts is not well constrained since only the CDF-N and CDF-S are capable of detecting these AGNs and their optical identification is quite challenging. Some progress has been made using type I AGNs selected in the soft X-ray band (Hasinger et al. 2005) and optically selected QSOs found in Lyman break galaxy surveys (Hunt et al. 2004) that clearly show a flatter faintend slope compared to lower redshifts.

Finally, we use a modified PLE (mod-PLE) function as described above and in Table 4 to allow us greater flexibility. Accounting for the flattening of the faint-end slope $(\alpha)$ without a dependence on the evolution ( $e 2)$ above the cutoff redshift may mitigate the problem mentioned above. The results shown in Figure 9 (solid line) are also consistent with our binned $1 / V_{a}$ measurements and show better agreement in the redshift interval $2<z<3$ than the LDDE fit. We find the dependence of the faint-end slope on redshift $\left(\alpha=-1.04_{-0.12}^{+0.11}\right)$ a bit stronger than that measured by Hopkins et al. $\left(2007 ; k_{\gamma 1}=-0.623 \pm 0.132\right)$. At higher redshifts $(z>3)$, this model may overestimate the numbers of low-luminosity AGNs with $\log L_{\mathrm{X}}<44$. This is evident by comparing the observed and expected numbers of highredshift AGNs in the deep Chandra fields (see $\S 8$ ). Interestingly, the difference between these two models below the break luminosity becomes larger at $z>4$ with $\sim 4$ times more AGNs with $\log L_{\mathrm{X}} \sim 43$. This region of $L_{\mathrm{X}}-z$ parameter space is primarily an extrapolation of the data. In $\S 8$ we investigate whether it is possible to accurately measure the faint-end slope at these high redshifts with current or future Chandra observations.

\section{MISSED SOURCES, TYPE 2 QSOs, AND THE COSMIC X-RAY BACKGROUND}

The intensity of the CXB, the integrated measure of the X-ray emission over all redshifts and luminosities, allows us to assess what fraction of the population we may be missing from our optically identified point sources in the $2-8 \mathrm{keV}$ band. In addition to constraining an undetected population of X-ray sources, we can also assess whether our incompleteness corrections to

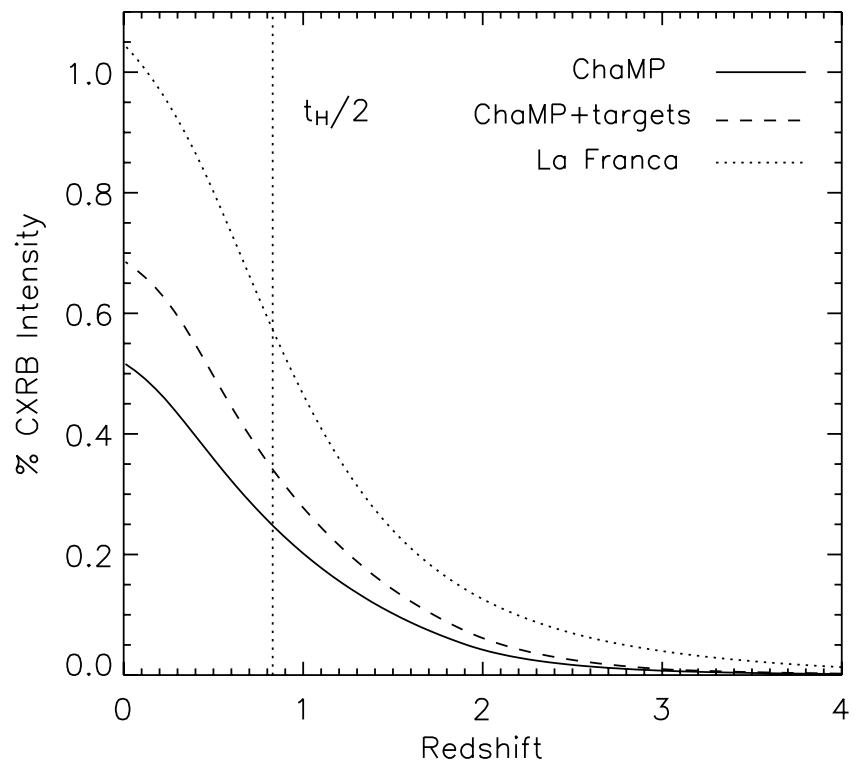

FIG. 14.-Cumulative fraction of the $2-8 \mathrm{keV} \mathrm{CXB}$ as a function of redshift. The solid line corresponds to our best-fit LDDE model (C), and the dashed line to a corrected version that accounts for excluded, faint $\left(r^{\prime}, i^{\prime}>24\right)$ targets. For comparison, we show (dotted line) the resolved fraction for the best-fit LDDE model of La Franca et al. (2005). The vertical line marks half a Hubble time.

account for unidentified sources are reasonable. The intensity of the CXB in the 2-8 keV band has been measured with several hard X-ray observatories over the last few decades (e.g., Moretti et al. 2003) and has been shown to have significant scatter ( $\sim 20 \%$ ) presumably due to instrument calibration. Here we compare to the best-fit value from Moretti et al. (2003) of $1.79 \times$ $10^{-11} \mathrm{ergs} \mathrm{cm}^{-2} \mathrm{~s}^{-1} \mathrm{deg}^{-2}$ in the $2-8 \mathrm{keV}$ band that has been converted from the $2-10 \mathrm{keV}$ band based on an assumed CXB spectrum with photon index of 1.4.

In Figure 14 we show the integrated contribution of AGNs from our best-fit LDDE model (C; solid line) to the CXB as a function of redshift. As shown, we account for $52 \%$ of the 2$8 \mathrm{keV} \mathrm{CXB}$ at $z=0$. We assess the contribution of AGNs that have X-ray fluxes above our limits but fell out of the sample due to their faint optical magnitudes $\left(r^{\prime}, i^{\prime}>24\right)$ by applying a correction based on the assumption that their redshift distribution is equivalent to identified sources at similar X-ray fluxes. These sources, as shown in the top panels of Figures 3 and 4 by the dotdashed line, mainly occupy the faint end of the X-ray flux distribution. Our measure of the resolved fraction of the CXB now reaches $68 \%$ (Fig. 14; dashed line). These results are not surprising since Worsley et al. (2005) find that $\sim 25 \%$ of the hard $\mathrm{CXB}$ remains to be resolved. This is also consistent with the recent resolved fraction of $79 \% \pm 8 \%$ measured in the $2-8 \mathrm{keV}$ band by Hickox \& Markevitch (2006). Both studies attribute the bulk of this unresolved emission to be from absorbed AGNs $\left(\log N_{\mathrm{H}} \sim\right.$ $23-24 \mathrm{~cm}^{-2}$ ) with redshift roughly between 0.5 and 1.5 . Therefore, at most, we may be inaccurately accounting for $\sim 10 \%$ of the AGN population due to our rather simple assumptions for the optically faint X-ray detections. Both Ueda et al. (2003) and La Franca et al. (2005) have measured resolved fractions close to unity. Other sources of uncertainty may be due to the fact that we did not correct our fluxes for X-ray absorption and excluded the targets of each ChaMP field for which a few are bright AGNs (Kim et al. 2007a). We further illustrate in Figure 14 that the hard CXB is mainly generated by AGNs at $z<1$, when the universe was more than half its present age, as previously described in many works to date (e.g., Barger et al. 2001). 
A current limitation of the ChaMP sample is the lack of absorbed $\left(N_{\mathrm{H}}>10^{22} \mathrm{~cm}^{-2}\right)$ and luminous $\left(\log L_{\mathrm{X}}>44\right)$ AGNs mainly due to the bright optical magnitude cut for optical spectroscopic follow-up (Silverman et al. 2005a; Green et al. 2004). The lack of these AGNs may not greatly alter our current luminosity function since they do not seem to outnumber the unabsorbed (i.e., type 1) AGNs at these high luminosities. Ueda et al. (2003) find the absorbed fraction at these luminosities to be $\sim 30 \%$, while La Franca et al. (2005) find an even lower fraction of $\sim 20 \%$. An accurate assessment is still required since the numbers of highly absorbed and luminous AGNs (i.e., type 2 QSOs) are still limited. The ChaMP project has initiated a deep optical spectroscopic campaign on Gemini to identify the optically faint X-ray sources (see Fig. 13 of Silverman et al. 2005a) that may provide a fair sample of these AGNs, thus removing our current selection bias. If we do relax our definition of a type II QSO to having FWHM $<2000 \mathrm{~km} \mathrm{~s}^{-1}$, as done by many studies (Szokoly et al. 2004; Eckart et al. 2006), we have found two such QSOs at $z>3$ (CXOMP J114036.2+661317, CXOMP J001756.6+163006) as shown in Figure 7. The SEXSI survey (Eckart et al. 2005, 2006; Harrison et al. 2003), with an unprecedented sample of 33 luminous AGNs lacking broad optical emission lines, has demonstrated that deep 8-10 m class spectroscopy is key to identify these optically faint AGNs.

\section{COMPARISON WITH OPTICALLY SELECTED AGNs}

It is useful to directly compare our XLF to the latest optical luminosity functions, as also done in recent works (e.g., Ueda et al. 2003; Barger et al. 2005; Richards et al. 2006) that cover a similar redshift range. Currently, the SDSS (Richards et al. 2006; Jiang et al. 2006) and COMBO-17 (Wolf et al. 2003), which solely detect type $1 \mathrm{AGNs}$, cover a redshift range out to $z \sim 5$. We elect to use cgs units throughout this work and transform the optical luminosity functions to match our XLF. We convert the luminosity used for the SDSS in units of absolute magnitude $\left(M_{i}\right.$; $z=2$ ) into a monochromatic luminosity $\left(l_{2500 \AA}\right)$ using the transformation given in equation (4) of Richards et al. (2006). The COMBO-17 rest-frame magnitudes $M_{1450}$ are converted to $l_{2500 \AA}$ assuming a power-law slope for the spectral energy distribution (SED; $f_{\nu} \propto \nu^{-\alpha}$ ) with spectral index ( $\alpha$ ) equal to 0.5 . These monochromatic rest-frame UV luminosities are translated into a monochromatic X-ray luminosity $\left(l_{2} \mathrm{keV}\right)$ using equation $(1 \mathrm{c})$ of Steffen et al. (2006). This relation has been shown by many X-ray to optical studies of AGNs (e.g., Wilkes et al. 1994; Green et al. 1995; Steffen et al. 2006) to have no redshift dependence and is applicable out to $z=5$ (Vignali et al. 2005). The slope of this relation is further supported, as shown by Ueda et al. (2003) by shifting the hard XLF to match the optical at lower redshifts $(z<2.3)$. We determine the rest-frame $2-8 \mathrm{keV}$ X-ray luminosity from $l_{2} \mathrm{keV}$ by assuming a power-law $\operatorname{SED}\left(L_{E} \propto E^{-\Gamma}\right)$ with $\Gamma=2.0$ (Reeves \& Turner 2000).

We show in Figure 15 a comparison of our XLF with the data and analytic model fits from the SDSS and COMBO-17 in the four highest redshift intervals mainly to highlight the behavior above and below the QSO peak at $z \sim 2.5$. For the optically selected luminosity functions, we limit the luminosity range to that which is covered by these surveys. This allows us to illustrate the complementarity of these surveys and avoid any interpretation based on extrapolations beyond the dynamic range of each sample. In general, the bright-end slope of our XLF agrees with that from the optical surveys out to $z \sim 5$, with the exception of the $1.5<z<2.0$ redshift interval, where the SDSS model is much steeper even compared to both X-rays and COMBO-17 (see Richards et al. 2006). Qualitatively, there does not appear to be any strong evidence for a change in the slope of the bright end with redshift (see Fig. 9 for a larger redshift baseline), as shown recently (Richards et al. 2006; Hopkins et al. 2007). At lower redshifts $(z<1.5)$, the slope at the bright end is not nearly as steep as measured in Richards et al. (2006) although larger samples of luminous X-ray-selected AGNs are required to justify such a statement. As shown, the faint end of the luminosity function can be probed by optically selected surveys (Jiang et al. 2006; Hunt et al. 2004) of type 1 AGNs at $z<3$. As expected, their space density falls below those found from X-ray selection due to the lack of obscured AGNs. At $z>3$, the luminosities of these optically selected AGNs do not fall well below the knee. As evident, X-ray surveys can probe moderate-luminosity AGNs at $3<z<4$, fainter than the break luminosity $\left(\log L_{\mathrm{X}}<44.5\right.$ ), thus allowing a measure of the slope at the faint end. Some uncertainty remains since the contribution of luminous type 2 QSOs is still uncertain, especially at $z>3$. However, as previously mentioned, recent constraints on the type 2 QSO population ( 25\%-50\%; Gilli et al. 2007) tend to show limited effect on our current measure of the XLF even when considering the possible increase in the obscured fraction with redshift (Treister \& Urry 2006) since the luminosity dependence is stronger than the redshift dependence.

\section{ACCRETION HISTORY OF SMBHs}

The luminosity function of AGNs is one of the key observational constraints on the mass buildup of SMBHs over cosmological time. With our measure of the XLF less biased against obscuration, sensitivity to lower luminosity AGNs, and coverage out to high redshift, we can reexamine, with very simplistic assumptions, our understanding of how the mass accretion rates, Eddington masses, and the cumulative mass density of SMBHs behave as a function of redshift. In addition, we can ascertain whether the well-known similarity between the accretion rate onto SMBHs and the star formation history of galaxies (Boyle \& Terlevich 1998; Franceschini et al. 1999; Merloni et al. 2004), from the QSO peak $(z \sim 2.5)$ to the present, persists at higher redshifts.

We determine the distribution of mass accretion rate as a function of redshift with the assumption that the accretion rate is directly proportional to the bolometric luminosity ( $L_{\text {bol }}$; eq. [12]) by a constant mass-to-energy conversion factor $\epsilon_{\text {rad }}$ :

$$
L_{\mathrm{bol}}=\epsilon_{\mathrm{rad}} \dot{M}_{\mathrm{acc}} c^{2} .
$$

We derive bolometric luminosities from the rest-frame $2-8 \mathrm{keV}$ $\mathrm{X}$-ray luminosity using the nonlinear relation given in equation (21) of Marconi et al. (2004) that mainly accounts for changes in the overall SED of AGNs as a function of optical luminosity (e.g., Steffen et al. 2006). We fix the accretion efficiency $\epsilon_{\text {rad }}$ to 0.1 (e.g., Marconi et al. 2004). In Figure 16 we plot the accretion rate distribution, using our best-fit LDDE model (C), at six redshifts that span the full extent of our XLF. Using the same bolometric luminosities, we label the top axis with the corresponding Eddington masses. We stress that these accretion rates and masses are highly degenerate. Here we aim to just illustrate the evolutionary behavior of the population in terms of their physical properties rather than present well-constrained physical measurements. In light of these words of caution, we see that the number density of the entire distribution systematically rises with cosmic time from $z=4.5$ to 2.5 (dotted line), which may represent the global ignition of mass accretion onto SMBHs at early epochs. At redshifts below this peak, the number density of AGNs with high accretion rates $(\log \dot{M} \gtrsim 1)$ or masses $\left(M_{\text {Edd }} \gtrsim 10^{8} M_{\odot}\right)$ 

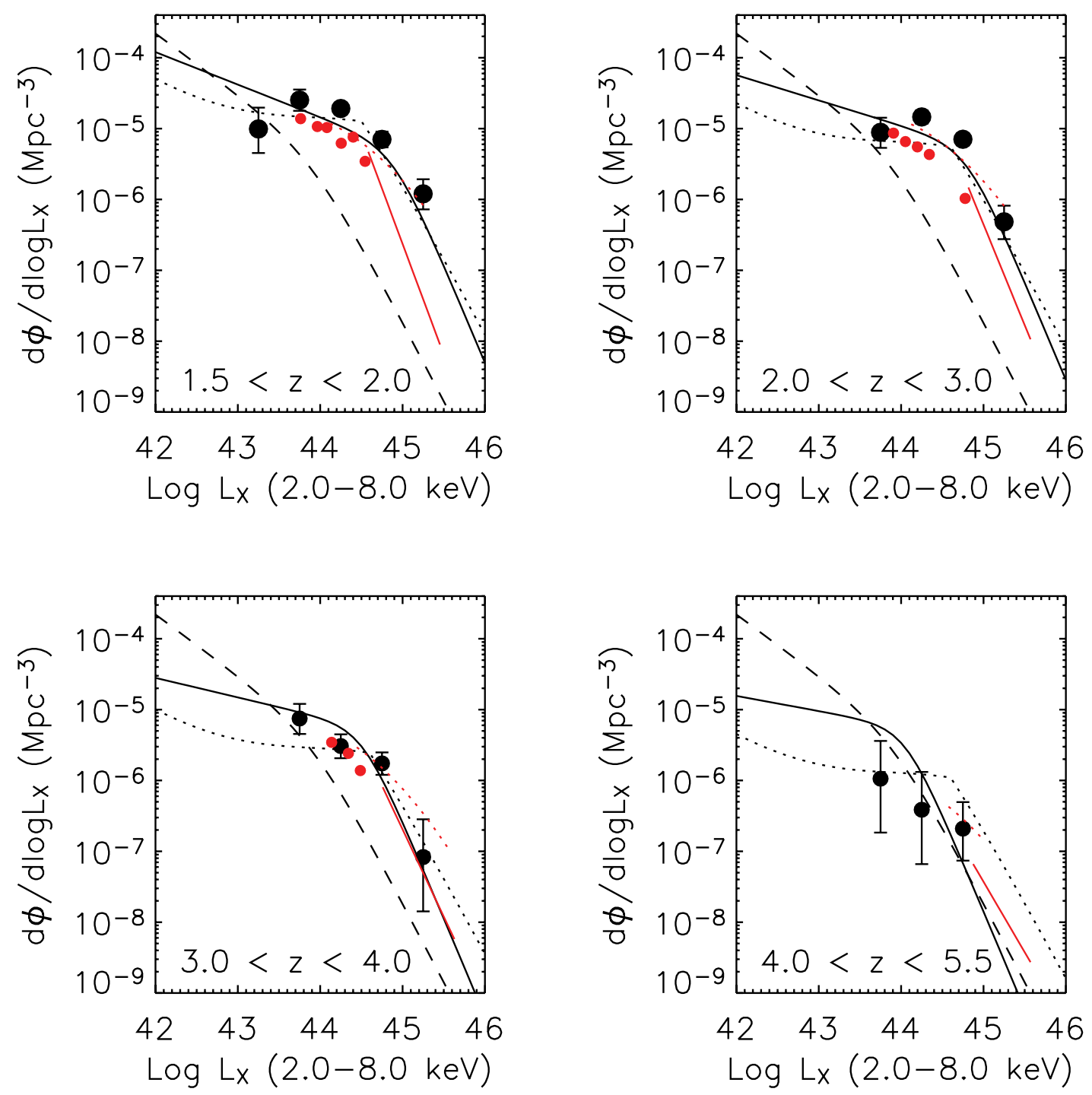

FIG. 15.-Comparison of the X-ray and optical luminosity functions at $z>1.5$. The black filled circles and lines are our data as shown in Fig. 9. The optical luminosity

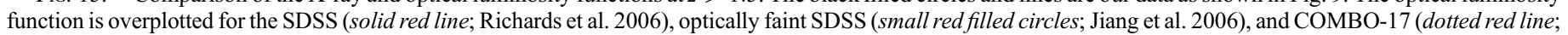
Wolf et al. 2003).

essentially halts to $z \sim 1.5$ and then declines rapidly. In contrast, the number of AGNs with low accretion rates $(\log \dot{M} \lesssim 1)$ or masses $\left(M_{\mathrm{Edd}} \lesssim 10^{7} M_{\odot}\right)$ continues to climb. We are witnessing either the emergence of young SMBHs (i.e., low mass, high accretion rate) or the remnants of a once highly luminous SMBH population (i.e., high mass, low accretion rate). Much recent evidence points to a luminosity-dependent lifetime (e.g., Hopkins et al. 2005; Adelberger \& Steidel 2005) that must also be folded in to accurately extract physical quantities from a luminosity function. Evidently, a more careful deconvolution is warranted.

As done in many recent studies, we can measure the cumulative mass density of SMBHs as a function of redshift from the integrated light from AGNs (Soltan 1982) using the same assumption for the radiative efficiency given above. In particular, the local value $(z=0)$ allows a direct comparison with complementary techniques (e.g., local galaxy luminosity function and a relation between black hole mass and its host bulge; integrated light from optically selected QSOs). With the drop-off in the number of AGNs at $z>3$, we do not expect our measure of the local value of the mass density to differ from recent estimates but aim to illustrate that further constraints on the mean growth of
AGNs at high redshifts are now possible. In Figure 17 we plot the results using our best-fit mod-PLE model (D) over an extended luminosity $\left(41<\log L_{2-8 \mathrm{keV}}<47\right)$ and redshift range $(0<z<$ 6 ). We do not account, as done in $\S 5$, for the optically faint X-ray sources not included in our sample; their contribution is not trivial to assess since their redshift distribution is most likely dissimilar to optically brighter AGNs at similar X-ray fluxes as demonstrated by Martinez-Sansigre et al. (2005). No correction for intrinsic absorption is applied. We have measured the mass density of black holes for two extreme values of the radiative accretion efficiency that is less constrained than the bolometric corrections involved. The gray area illustrates the range of mass densities, with values of the accretion efficiency ranging from a Schwarzschild $\left(\epsilon_{r}=0.06\right)$ to a $\operatorname{Kerr}\left(\epsilon_{r}=0.3\right)$ black hole. The solid line gives the values for the widely accepted value $\epsilon=0.1$ with a mass density at $z=0$ of $1.64 \times 10^{5} M_{\odot} \mathrm{Mpc}^{-3}$. In addition, we show the value for a constant bolometric correction (40; Elvis et al. 1994) with $\epsilon=0.1$. Our estimate of the local mass density in SMBHs is lower than other measures (left panel). Our value is $48 \%$ of the local value, determined from the galaxy luminosity function and velocity dispersion, of $3.4_{-0.5}^{+0.6} \times 10^{5} M_{\odot} \mathrm{Mpc}^{-3}$ 


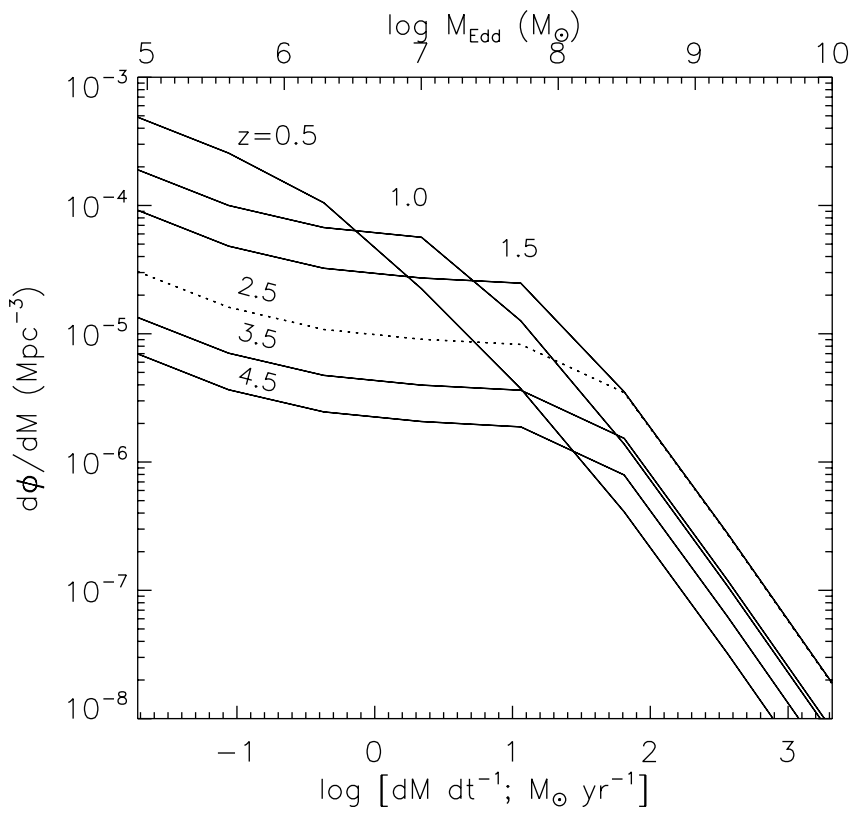

FIG. 16.-Distribution of mass accretion rate onto SMBHs at redshifts above and below the peak number density $(z=2.5$; dashed line $)$ using our best-fit LDDE model (C) and a fixed accretion efficiency. The top axis shows the Eddington masses assuming an equivalent bolometric luminosity.

(Marconi et al. 2004) and is in closer agreement to the values obtained from previous hard X-ray-selected AGNs (Barger et al. 2005; La Franca et al. 2005; Ueda et al. 2003). In particular, Barger et al. (2005) find a similar local black hole mass density $\left(2.1 \times 10^{5} M_{\odot} \mathrm{Mpc}^{-3}\right)$ when using a bolometric correction factor (35) close to that employed here. Barger et al. (2005) point out that the mass density measured by Yu \& Tremaine (2002) is possibly too high due to an extrapolation of the optical luminosity function at the faint end that has now been shown to be shallower (Croom et al. 2004). The fact that we are not accounting for optically faint X-ray sources, resolving $\sim 70 \%$ of the hard CXB, and do not account for Compton-thick AGNs could contribute to the discrepancy with mass density estimates using local galaxies. As is evident in the right panel of Figure 17, most (81\%) of the growth of SMBHs occurred by the time the universe was half $(z \sim 0.7)$ its present age, with minimal contribution at $z>3$.

\subsection{Coevolution of SMBHs and Star-forming Galaxies}

The order-of-magnitude decline in the comoving mean emissivity of AGNs and star formation history of galaxies from $z \sim$ 1.5 to the present has initiated many early postulates for a coevolution scheme of SMBHs and the galaxies in which they reside. Boyle \& Terlevich (1998) demonstrated that the optical QSO luminosity function exhibited a similar behavior to the SFR of field galaxies. Franceschini et al. (1999), with an X-rayselected sample of AGNs, further investigated this correlation by demonstrating that the more luminous QSOs evolve closely with the most massive galaxies (i.e., E/S0) while the lower luminosity AGNs track the star-forming, field galaxy population. At the time, these studies were mainly limited to $z<3$ due to the availability of measured SFRs of galaxies.

Armed with our XLF of AGNs out to $z \sim 5$ and the latest SFRs of high-redshift galaxies, we can revisit this correlation. In the last few years, new samples of high-redshift galaxies have enabled the evolution of the SFR to be extended out to $z \sim 6$ (e.g., Bunker et al. 2004; Giavalisco et al. 2004; Bouwens et al. 2007). Most studies have selected these high-redshift galaxies by Lyman break techniques (e.g., Steidel et al. 1999) or narrow emission line searches (e.g., Rhoads et al. 2003). With the advent of wide-area, multislit spectrographs on 8-10 m class telescopes, flux-limited surveys are able to probe a sizeable volume and identify significant numbers of high-redshift galaxies without preset color selection criteria or emission-line strengths (Le Fèvre et al. 2005).

We have measured the mass accretion rate per comoving volume (units of $M_{\odot} \mathrm{yr}^{-1} \mathrm{Mpc}^{-3}$ ) as a function of redshift using our

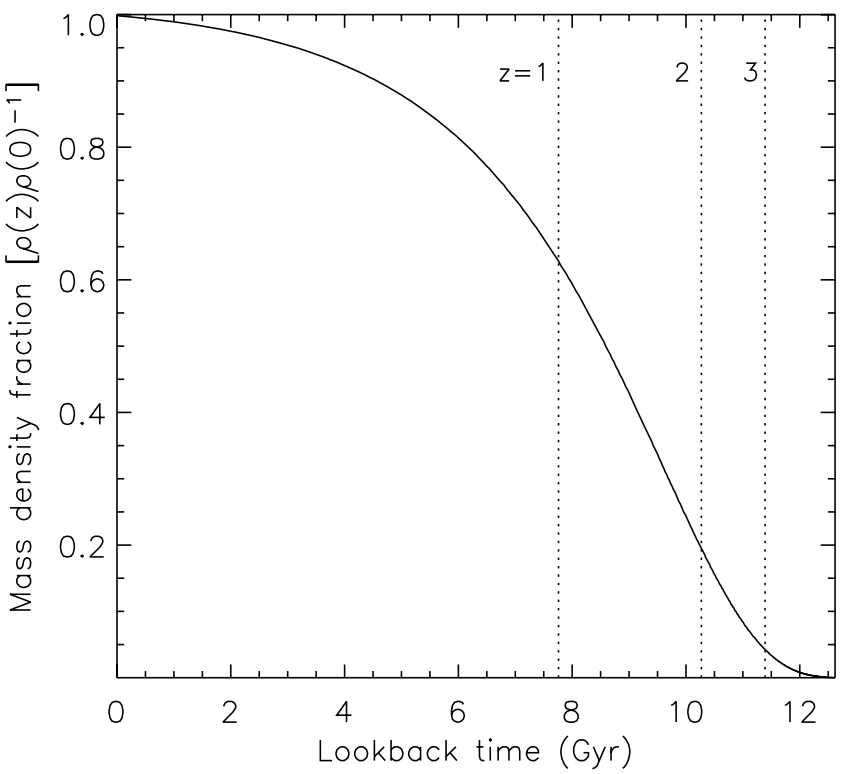

FIG. 17.- Integrated mass density of SMBHs. Left: The dark gray region shows our cumulative values using our best-fit mod-PLE model (D), a luminosity-dependent bolometric correction, and a range of accretion efficiencies of $0.06<\epsilon_{r}<0.3$, with a solid line marking the $\epsilon_{r}=0.1$ case. We plot with a dashed line the results using a constant bolometric correction and $\epsilon_{r}=0.1$. For comparison, we have highlighted the $z=0$ mass densities (horizontal dashed lines) measured using the optical QSO luminosity function (Yu \& Tremaine 2002) and hard XLF (Marconi et al. 2004) of Ueda et al. (2003). The dotted line marks the mass density as a function of redshift using the hard XLF of La Franca et al. (2005). Right: We show the cumulative mass density, identical to the case on the left marked by a solid line, as a function of look-back time normalized by our local value. 


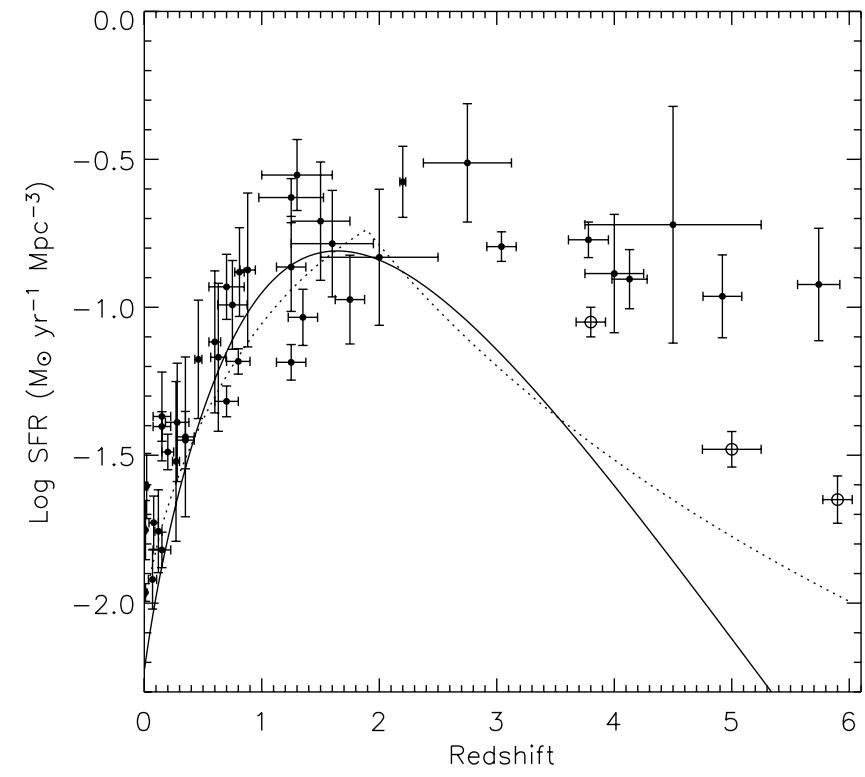

FIG. 18.-Comparison of the star formation history of galaxies (data points) to the mass accretion rate $\left(M_{\odot} \mathrm{yr}^{-1} \mathrm{Mpc}^{-3}\right)$ of SMBHs using the mod-PLE (solid line) and LDDE (dotted line) models. SFRs from the compilation of Hopkins (2004) are marked by the small filled circles, while the open circles are recent measurements at $z \gtrsim 4$ (Bouwens et al. 2007). The mass accretion rates have been scaled up by a factor of 5000. The divergence at $z>4$ of the two lines representing the accretion history of SMBHs highlights the poor constraints on the XLF at early epochs.

analytic models (C, D) for all AGNs with $L_{2-8 \mathrm{keV}}>10^{42} \mathrm{ergs} \mathrm{s}^{-1}$. Bolometric corrections and conversion factors to accretion rates are used as described in the previous section. In Figure 18 we plot the results compared to the dust-corrected SFRs ( filled circles) as compiled by Hopkins (2004). We add the latest measurements at $z \gtrsim 4$ from Bouwens et al. (2007) as shown by the open circles. The mass accretion rates have been scaled up by a factor of 5000 . Up to $z \sim 2$, there is an overall similarity as previously elaborated in many studies to date. The discrepancy noted by Haiman et al. (2004) at $z<2$ appears to be removed with the inclusion of obscured AGNs from X-ray-selected surveys. The peak in the black hole accretion rate density now occurs at $z \sim 1.5$ rather than $z \sim 2.5$ as measured with optically selected QSO samples. Also noted in many investigations, there is a divergence at $z>2$ between the two growth rates, with a faster decline of the AGN population even when comparing to the recent SFRs (Bouwens et al. 2007) that show a significant decline between $4<z<6$. Here we find a decrease in the accretion rate onto SMBHs by a factor of 3-4 compared to the SFRs at $z \sim 4.5$. This may represent a delay between the formation of stars and fully matured
SMBHs in the early universe possibly due to either the cooling times required for gas to be available for accretion or an insufficient merger history (for further discussion see Haiman et al. 2004).

\section{DETECTION OF HIGH-REDSHIFT AGNs IN CHANDRA SURVEYS}

Even though we have extended the $2-8 \mathrm{keV}$ luminosity function beyond $z \sim 3$, there remains much uncertainty about the behavior of the luminosity function below the break in the $3<$ $z<4$ redshift range and at all luminosities at higher redshifts. Since Chandra has now completed or will undertake some new surveys that cover a wider area and at moderate depths, we can make some predictions of their return of high-redshift AGNs. We have computed the numbers of AGNs in two redshift intervals and two luminosity ranges that basically span below $(42<$ $\left.\log L_{\mathrm{X}}<44\right)$ and above $\left(44<\log L_{\mathrm{X}}<46\right)$ the break luminosity. We give these values for various surveys using our best-fit LDDE and mod-PLE (in parentheses) models in Table 5. The estimates between the two models are similar at high luminosities but differ at the low-luminosity end, which becomes even more pronounced at the highest redshifts (see Fig. 9).

We see that the ChaMP will provide a total of $\sim 46$ AGNs at $z>3$ based on an area coverage of $2 \mathrm{deg}^{2}$ that is similar to the numbers expected from the latest wider area surveys (i.e., EGS, Chandra/COSMOS). As mentioned earlier, these surveys are complementary to ChaMP since they reach greater depths over a smaller area to detect AGNs at lower luminosities as exemplified here. We note that the mod-PLE model is most likely overpredicting the numbers of lower luminosity $\left(\log L_{\mathrm{X}}<44\right)$ AGNs based on the observed number in the CDF-N and CDF-S. In total, we expect a final sample of over $\sim 195$ AGNs at $z>3$ with 63 above a redshift of 4 . Better constraints on the faint end of the luminosity function in the $3<z<4$ redshift interval can be achieved by either the E-CDF-S, EGS, or cCOSMOS with a sample of $\sim 15-25$ sources per survey, although an accurate measure of the slope will require the combined sample that is especially true at higher redshifts. An accurate assessment of the luminosity function at $z>4$ will definitely require a merger of all available catalogs.

\section{SUMMARY}

We have presented an extension of the hard (2-8 keV) XLF of AGNs up to $z \sim 5$. The ChaMP effectively covers a wide area $\left(1.8 \mathrm{deg}^{2}\right)$ at sufficient depths $\left(f_{0.5-2.0 \mathrm{keV}} \sim 10^{-15} \mathrm{ergs} \mathrm{cm}^{-2} \mathrm{~s}^{-1}\right)$ to significantly improve the statistics of luminous $\left(L_{\mathrm{X}}>10^{44} \mathrm{ergs}\right.$ $\mathrm{s}^{-1}$ ) AGNs at high redshift. In total, we have amassed a sample of 682 AGNs with 31 at $z>3$. The addition of lower luminosity AGNs from the Chandra Deep Fields is instrumental to

TABLE 5

Predicted Number of High-Redshift AgNs in Complete Surveys

\begin{tabular}{|c|c|c|c|c|c|}
\hline \multirow[b]{2}{*}{ SURVEY } & \multicolumn{2}{|c|}{$3<z<4$} & \multicolumn{2}{|c|}{$4<z<6$} & \multirow[b]{2}{*}{ TOTAL } \\
\hline & $\log L_{\mathrm{X}}<44$ & $\log L_{X}>44$ & $\log L_{\mathrm{X}}<44$ & $\log L_{\mathrm{X}}>44$ & \\
\hline ChaMP ................................... & $3(6)$ & $31(41)$ & $0(0)$ & $12(6)$ & $46(53)$ \\
\hline 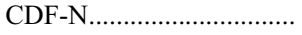 & $4(16)$ & $3(5)$ & $2(9)$ & $2(2)$ & $11(32)$ \\
\hline CDF-S & $4(12)$ & $3(4)$ & $2(6)$ & $2(2)$ & $11(24)$ \\
\hline 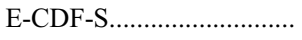 & $11(35)$ & $8(13)$ & $4(18)$ & $6(5)$ & $29(71)$ \\
\hline EGS & $12(38)$ & $16(25)$ & $4(13)$ & $11(8)$ & $43(84)$ \\
\hline cCOSMOS …....................... & $15(45)$ & $22(35)$ & $4(14)$ & $14(12)$ & $55(106)$ \\
\hline Totals ................................ & $49(152)$ & 73 (114) & $16(60)$ & $47(35)$ & $195(370)$ \\
\hline
\end{tabular}


characterize the faint-end slope at $z \gtrsim 1$. We have corrected for incompleteness (i.e., fraction of sources without a redshift) as a function of both X-ray flux and optical magnitude, as dictated by the limitations of optical spectroscopic follow-up. Significant optical follow-up is still required to accurately account for the obscured population, especially at high redshifts.

We have measured the hard XLF with both a binned $\left(1 / V_{a}\right)$ and an unbinned method that fits an analytic model to our data using a maximum likelihood technique. We found that the luminosity function is similar to that found in previous studies (Ueda et al. 2003; La Franca et al. 2005; Barger et al. 2005) up to $z=3$, with an evolution dependent on luminosity. At higher redshifts, there is a significant decline in the numbers of AGNs with an evolution rate similar to that found with optically selected QSO samples. We further show that the strong evolution above the cutoff redshift may cause the LDDE model to underpredict the number of low-luminosity AGNs at $z>2$. A PLE model with a faint-end slope dependent on redshift agrees better with the binned $\left(1 / V_{a}\right)$ data at $z \sim 2.5$ although it may overpredict the number of faint AGNs at higher redshifts. We highlight the need to improve the statistics of both high-redshift AGNs at $z>4$ and lower luminosity (i.e., below the break in the luminosity function) AGNs at $z>3$. Such improvements are feasible from our predictions of the numbers of AGNs that will be found in the latest Chandra surveys (E-CDF-S, EGS, and COSMOS).
Our new luminosity function accounts for $\sim 52 \%$ of the $2-8 \mathrm{keV}$ $\mathrm{CXB}$. The integrated emission from these AGNs gives a $z=0$ mass density of SMBHs of $1.64 \times 10^{5} M_{\odot} \mathrm{Mpc}^{-3}$, lower than other published values using X-ray-selected AGN samples and the local value measured using a galaxy luminosity function and a bulge-velocity relation, possibly due to unaccounted optically faint AGNs $\left(r^{\prime}, i^{\prime}>24\right)$ and Compton-thick AGNs. Further, a comparison of the mean mass accretion rate of SMBHs to the star formation history of galaxies out to $z \sim 5$ shows the familiar coevolution scheme up to $z \sim 2$ and a divergence at higher redshifts, with perhaps star formation preceding the formation of SMBHs.

The authors wish to thank the NOAO and SAO telescope allocation committees for their support of this work. We also recognize the contributions from the following people for either their insightful discussions, general comments on the work, or providing electronic tables such as the XMM-Newton data: Dave Alexander, Hermann Brunner, Jacobo Carrero, Fabio La Franca, Vincenzo Mainieri, Israel Matute, Andrea Merloni, Takamitsu Miyaji, Francesco Shankar, and Gyula Szokoly.

Facilities: CXO, Mayall, Blanco, WIYN, Magellan:Baade, Magellan:Clay, MMT, FLWO:1.5m
Adelberger, K. L., \& Steidel, C. C. 2005, ApJ, 630, 50

Akiyama, M., Ueda, Y., Ohta, K., Takahashi, T., \& Yamada, T. 2003, ApJS, 148,275

Alexander, D. M., Smail, I., Bauer, F., Chapman, S. C., Blain, A. W., Brandt, W. N., \& Ivison, R. J. 2005, Nature, 434, 738

Alexander, D. M., et al. 2003, AJ, 126, 539

Appenzeller, I., et al. 1998, ApJS, 117, 319

Arnouts, S., et al. 2001, A\&A, 379, 740

Avni, Y., \& Bahcall, J. N. 1980, ApJ, 235, 694

Barger, A. J., Cowie, L. L., Mushotzky, R., \& Richards, E. A. 2001, AJ, 121, 662

Barger, A. J., Cowie, L. L., Mushotzky, R. F., Yang, Y., Wang, W.-H., Steffen, A. T., \& Capak, P. 2005, AJ, 129, 578

Barger, A. J., et al. 2003a, AJ, 126, 632

2003b, ApJ, 584, L61

Barkhouse, W. A., et al. 2006, ApJ, 645, 955

Binggeli, B., Sandage, A., \& Tammann, G. A. 1988, ARA\&A, 26, 509

Bouwens, R. J., Illingworth, G. D., Franx, M., \& Ford, H. 2007, ApJ, 670, 928

Bower, R. G., et al. 1996, MNRAS, 281, 59

Boyle, B. J., Shanks, T., \& Peterson, B. A. 1988, MNRAS, 235, 935

Boyle, B. J., \& Terlevich, R. J. 1998, MNRAS, 293, L49

Brandt, W. N., Guainazzi, M., Kaspi, S., Fan, X., Schneider, D. P., Strauss, M. A., Clavel, J., \& Gunn, J. E. 2001a, AJ, 121, 591

Brown, M., et al. 2006, ApJ, 638, 88

Brunner, H., Cappelluti, N., Hasinger, G., Barcons, X., Fabian, A., Mainieri, V., \& Szokoly, G. 2008, A\&A, 479, 283

Bunker, A., Stanway, E., Ellis, R., \& McMahon, R. G. 2004, MNRAS, 355 , 374

Capak, P., et al. 2004, AJ, 127, 180

Cavaliere, A., \& Vittorini, V. 2000, ApJ, 543, 599

Chapman, S. C., Blain, A. W., Smail, I., \& Ivison, R. J. 2005, ApJ, 622, 772

Cowie, L. L., Barger, A. J., Bautz, M. W., Brandt, W. N., \& Garmire, G. P. 2003, ApJ, 584, L57

Croom, S. M., Smith, R. J., Boyle, B. J., Shanks, T., Miller, L., Outram, P. J., \& Loaring, N. S. 2004, MNRAS, 349, 1397

della Ceca, R., Maccacaro, T., Gioia, I. M., Wolter, A., \& Stocke, J. T. 1992, ApJ, 389, 491

Eckart, M. E., Laird, E. S., Stern, D., Mao, P. H., Helfand. D. J., \& Harrison, F. A. 2005, ApJS, 156, 35

Eckart, M. E., Stern, D., Helfand, D., Harrison, F., Mao, P., \& Yost, S. A. 2006, ApJS, 165, 19

Elvis, M., et al. 1994, ApJS, 95, 1

Fan, X., et al. 2001, AJ, 121, 54 2004, AJ, 128, 515
Fiore, F., et al. 2003, A\&A, 409, 79

Franceschini, A., Hasinger, G., Miyaji, T., \& Malquori, D. 1999, MNRAS, 310, L5

Fukugita, M., Ichikawa, T., Gunn, J. E., Doi, M., Shimasaku, K., \& Schneider, D. P. 1996, AJ, 111, 1748

Giacconi, R., et al. 2002, ApJS, 139, 369

Giavalisco, M., et al. 2004, ApJ, 600, L93

Gilli, R., Comastri, R., \& Hasinger, G. 2007, A\&A, 463, 79

Gioia, I. M., Maccacaro, T., Schild, R. E., Wolter, A., Stocke, J. T., Morris, S. L., \& Henry, J. P. 1990, ApJS, 72, 567

Green, P. J., et al. 1995, ApJ, 450, 51 2004, ApJS, 150, 43

Haiman, Z., Ciotti, L., \& Ostriker, J. 2004, ApJ, 606, 763

Hao, L., et al. 2005, AJ, 129, 1783

Harrison, F. A., Eckart, M. E., Mao, P. H., Helfand, D. J., \& Stern, D. 2003, ApJ, 596, 944

Hasinger, G., Takamitsu, M., \& Schmidt, M. 2005, A\&A, 441, 417

Hasinger, G., et al. 2001, A\&A, 365, L45 2007, ApJS, 172, 29

Heckman, T. M., Kauffmann, G., Brinchmann, J., Charlot, S., Tremonti, C., \&

White, S. 2004, ApJ, 613, 109

Hewett, P. C., Foltz, C. B., \& Chaffee, F. H. 1993, ApJ, 406, L43

Hickox, R. C., \& Markevitch, M. 2006, ApJ, 645, 95

Hogg, D. W. 1999, preprint (astro-ph/9905116)

Hopkins, A. M. 2004, ApJ, 615, 209

Hopkins, P. F., Hernquist, L., Cox, T. J., Di Matteo, T., Robertson, B., \& Volker, S. 2005, ApJ, 630, 705

Hopkins, P. F., Richards, G. T., \& Hernquist, L. 2007, ApJ, 654, 731

Hunt, M. P., Steidel, C. C., Adelberger, K., \& Shapley, A. 2004, ApJ, 605, 625 James, F. 1994, MINUIT Reference Manual, CERN Program Library Writeup D506 (Geneva: CERN)

Jiang, L., et al. 2006, AJ, 131, 2788

Kartaltepe, J. S., et al. 2007, ApJS, 172, 320

Kim, D.-W., et al. 2004a, ApJ, 600, 59 2004b, ApJS, 150,19

2006, ApJ, 644, 829

Kim, M., Wilkes, B. J., Kim, D. W., Green, P. J., Barkhouse, W. A., Lee, M. G.,

Silverman, J. D., \& Tananbaum, H. D. 2007a, ApJ, 659, 29

Kim, M., et al. 2007b, ApJS, 169, 401

La Franca, F., et al. 2005, ApJ, 635, 864

Lampton, M., Margon, B., \& Bowyer, S. 1976, ApJ, 208, 177

Le Fèvre, O., et al. 2005, Nature, 437, 519

Lehmann, I., et al. 2001, A\&A, 371, 833

Lehmer, B. D., et al. 2005, ApJS, 161, 21 
Maccacaro, T., della Ceca, R., Gioia, I. M., Morris, S. L., Stocke, J. T., \& Wolter, A. 1991, ApJ, 374, 117

Mainieri, V., et al. 2002, A\&A, 393, 425 2005, A\&A, 437, 805

Marconi, A., Risaliti, G., Gilli, R., Hunt, L. K., Maiolino, R., \& Salvati, M. 2004, MNRAS, 351, 169

Marshall, H. L. 1987, AJ, 94, 628

Marshall, H. L., Tananbaum, H., Avni, Y., \& Zamorani, G. 1983, ApJ, 269, 35 Martinez-Sansigre, A., Rawlings, S., Lacy, M., Fadda, D., Marleau, F. R., Simpson, C., Willott, C. J., \& Jarvis, M. 2005, Nature, 436, 666

Mason, K. O., et al. 2000, MNRAS, 311, 456

McHardy, I., et al. 1998, MNRAS, 295, 641

Merloni, A. 2004, MNRAS, 353, 1035

Merloni, A., Rudnick, G., \& Di Matteo, T. 2004, MNRAS, 354, L37

Miyaji, T., Hasinger, G., \& Schmidt, M. 2000, A\&A, 353, 25 2001, A\&A, 369, 49

Moretti, A., Campana, S., Lazzati, D., \& Tagliaferri, G. 2003, ApJ, 588, 696

Murray, S. S., et al. 2005, ApJS, 161, 1

Nandra, K., et al. 2005, MNRAS, 356, 568

Osmer, P. S. 1982, ApJ, 253, 28

Page, M. J., \& Carrera, F. J. 2000, MNRAS, 311, 433

Polletta, M., et al. 2006, ApJ, 642, 673

Reeves, J. N., \& Turner, M. J. L. 2000, MNRAS, 316, 234

Rhoads, J. E., et al. 2003, AJ, 125, 1006

Richards, G. T., Strauss, M. A., \& Fan, X. 2006, AJ, 131, 2766

Richards, G. T., et al. 2005, MNRAS, 360, 839

Rosati, P., et al. 2002, ApJ, 566, 667

Schmidt, M. 1968, ApJ, 151, 393

Schmidt, M., \& Green, R. 1983, ApJ, 269, 352

Schmidt, M., Schneider, D. P., \& Gunn, J. E. 1995, AJ, 110, 68

Schwope, A., et al. 2000, Astron. Nachr., 321, 1

Silverman, J. D. 2004, Ph.D. thesis, Univ. Virginia
Silverman, J. D., et al. 2002, ApJ, 569, L1 2003, Astron. Nachr., 324, 97 2005a, ApJ, 618, 123 2005b, ApJ, 624, 630

Smith, J. A., et al. 2002, AJ, 123, 2121

Soltan, A. 1982, MNRAS, 200, 115

Steffen, A. T., Barger, A. J., Capak, P., Cowie, L. L., Mushotzky, R. F., \& Yang, Y. 2004, AJ, 128, 1483

Steffen, A. T., Strateva, I., Brandt, W. N., Alexander, D. M., Koekemoer, A. M., Lehmer, B. D., Schneider, D. P., \& Vignali, C. 2006, AJ, 131, 2826

Steidel, C. C., Adelberger, K. L., Giavalisco, M., Dickinson, M., \& Pettini, M. 1999, ApJ, 519, 1

Szokoly, G. P., et al. 2004, ApJS, 155, 271

Tozzi, P., et al. 2001, ApJ, 562, 42

Treister, E., \& Urry, C. M. 2006, ApJ, 652, L79

Treister, E., et al. 2005, ApJ, 621, 104

Ueda, Y., Akiyama, M., Ohta, K., \& Takamitsu, M. 2003, ApJ, 598, 886

Vignali, C., Brandt, W. N., Schneider, D. P., \& Kaspi, S. 2005, AJ, 129, 2519

Wall, J. V., Jackson, C. A., Shaver, P. A., Hook, I. M., \& Kellermann, K. I. 2005, A\&A, 434, 133

Warren, S. J., Hewett, P. C., \& Osmer, P. S. 1994, ApJ, 421, 412

Wilkes, B. J., Tananbaum, H., Worrall, D., Avni, D. M., Oey, M. S., \& Flanagan, F. 1994, ApJS, 92, 53

Wolf, C., Wisotzki, L., Borch, A., Dye, S., Kleinheinrich, M., \& Meisenheimer, K. 2003, A\&A, 408, 499

Wolf, C., et al. 2004, A\&A, 421, 913

Worsley, M. A., et al. 2005, MNRAS, 357, 1281

Yang, Y., Mushotzky, R. F., Steffen, A. T., Barger, A. J., \& Cowie, L. L. 2004, AJ, 128, 1501

Yu, Q., \& Tremaine, S. 2002, MNRAS, 335, 965

Zamorani, G., et al. 1999, A\&A, 346, 731

Zheng, W., et al. 2004, ApJS, 155, 73 